\title{
Predictive Scenario for Premixed Methane-Air Flame Spreading and Explosion Triggering in a Mining Passage
}

\author{
Sri Hari Ramakrishna Chalagalla
}

Follow this and additional works at: https://researchrepository.wvu.edu/etd

\section{Recommended Citation}

Chalagalla, Sri Hari Ramakrishna, "Predictive Scenario for Premixed Methane-Air Flame Spreading and Explosion Triggering in a Mining Passage" (2015). Graduate Theses, Dissertations, and Problem Reports. 5333.

https://researchrepository.wvu.edu/etd/5333

This Thesis is protected by copyright and/or related rights. It has been brought to you by the The Research Repository @WVU with permission from the rights-holder(s). You are free to use this Thesis in any way that is permitted by the copyright and related rights legislation that applies to your use. For other uses you must obtain permission from the rights-holder(s) directly, unless additional rights are indicated by a Creative Commons license in the record and/ or on the work itself. This Thesis has been accepted for inclusion in WVU Graduate Theses, Dissertations, and Problem Reports collection by an authorized administrator of The Research Repository @ WVU. For more information, please contact researchrepository@mail.wvu.edu. 


\title{
Predictive Scenario for Premixed Methane-Air Flame Spreading and Explosion Triggering in a Mining Passage
}

\author{
Sri Hari Ramakrishna Chalagalla
}

Thesis submitted to the

Benjamin M. Statler College of Engineering and Mineral Resources

at

West Virginia University

in Partial Fulfillment of the Requirements for the Degree of

\author{
Master of Science \\ in \\ Mechanical Engineering \\ V'yacheslav Akkerman, Ph.D., Chair \\ Arvind Thiruvengadam, Ph.D. \\ Ali. S. Rangwala, Ph.D. \\ Department of Mechanical and Aerospace Engineering \\ Morgantown, West Virginia
}

(2015)

Keywords: fire safety; mining fire scenario; combustion instability; flame acceleration; detonation triggering; turbulent burning; dusty-gaseous combustion.

Copyright (2015) Sri Hari Ramakrishna Chalagalla 


\section{ABSTRACT}

To reveal the inner mechanism of gas explosion, the entire scenario of premixed flame front evolution within an accidental fire is prescribed, quantitatively, with the situation of a methane-air explosion in a mining passage as the primary application. Specifically, the key stages of flame evolution are scrutinized. First, a globally-spherical expansion of a centrally-ignited, embryonic flame, with a possibility of self-similar acceleration caused by the hydrodynamic (DarrieusLandau) instability occurs. This stage provides an order of magnitude increase in the flame speed in realistically large mining passages. Second, a transition from a globally-spherical front to a finger-shaped one happens, when a flame starts approaching the passage walls. While this acceleration is extremely strong, it stops as soon as the flame touches the passage wall. This mechanism is Reynolds-independent; being equally relevant to micro-channels and realistically large tunnels. The flame speed increases by one more order of magnitude during this stage. Eventually, a flame may accelerate due to wall friction as well as in-built obstacles and wall roughness. While this scenario could be dominant at micro- and mesa-scales, it appears negligible in a mining passage because the influence of wall friction decreases, drastically, with the Reynolds number, and wall-attached obstacles are small in mines. Overall, we have identified the key characteristics of all stages such as the timing for each stage as well as the flame shapes, propagation speeds, acceleration rates, and flame-generated velocity profiles. The flame speed rises by orders of magnitude. Starting with laminar homogenously-gaseous combustion, the

analysis is subsequently extended to dusty-gaseous environments. For this purpose, the dependences of the thermal-chemical flame parameters, such as the planar flame speed, versus the combustible and inert dust properties, such as the dust particles size and concentration, are incorporated into the formulation. 


\section{Acknowledgements}

I would like to express my gratitude to several key persons who have contributed towards the completion of this thesis and have influenced my professional development as a researcher.

First and foremost, I would like to thank Dr. Vyacheslav Akkerman, who initially gave me the opportunity to pursue my educational career at WVU, and continuously provided me with his huge support and guidance throughout my time here. Also, I sincerely thank my other committee members, Dr. Ali Rangwala and Dr. Arvind Thiruvengadam, for their valuable time and suggestions.

In addition, I would like to thank Dr. Vitaly Bychkov, who supported me with his suggestions for my research work.

Many friends have helped me stay sane through these difficult years. Their support and care helped me overcome setbacks and stay focused on my graduate study. I greatly value their friendship and I deeply appreciate their belief in me. I am also grateful to the Indian community that helped me adjust to a new country.

Most importantly, none of this would have been possible without the love and patience of my family. My immediate family, to whom this Thesis is dedicated to, has been a constant source of love, concern, support and strength all these years. I would like to express my heart-felt gratitude to my family.

I have to give a special mention for the support given by our team - Sinan Demir, Berk Demirgok, Orlando Ugarte, Serdar Bilgili, and Jad Sadek. I warmly appreciate the generosity and understanding of my colleagues.

Finally, I appreciate the financial support from The Alpha Foundation for the Improvement of Mine Safety and Health, WVU's (PSCoR) Program and WVU's Senate Research Grant that funded parts of the research discussed in this Thesis. 
I dedicate this thesis to my parents Satyanarayana Chalagalla and Drowpadi Chalagalla. 


\section{Table of Contents}

$\begin{array}{llllllllllll}\text { Abstract } & \ldots & \ldots & \ldots & \ldots & \ldots & \ldots & \ldots & \ldots & \ldots & \text { ii }\end{array}$

$\begin{array}{llllllllllll}\text { Acknowledgments } & \ldots & \ldots & \ldots & \ldots & \ldots & \ldots & \ldots & \ldots & \ldots & \text { iii }\end{array}$

$\begin{array}{llllllllllll}\text { Table of Contents } & \ldots & \ldots & \ldots & \ldots & \ldots & \ldots & \ldots & \ldots & \ldots & \mathrm{V}\end{array}$

$\begin{array}{llllllllllll}\text { Nomenclature } & \ldots & \ldots & \ldots & \ldots & \ldots & \ldots & \ldots & \ldots & \ldots & \text { vi }\end{array}$

$\begin{array}{lllllllllll}\text { List of Tables } & \ldots & \ldots & \ldots & \ldots & \ldots & \ldots & \ldots & \ldots & \ldots & \text { ix }\end{array}$

$\begin{array}{llllllllllll}\text { List of Figures } & \ldots & \ldots & \ldots & \ldots & \ldots & \ldots & \ldots & \ldots & \ldots & \mathrm{X}\end{array}$

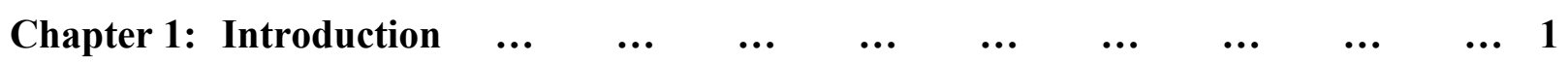

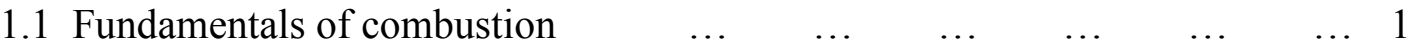

$\begin{array}{llllllllll}1.2 & \text { Mining fire and explosion } & \ldots & \ldots & \ldots & \ldots & \ldots & \ldots & \ldots & 2\end{array}$

$\begin{array}{llllllllllll}1.3 & \text { Objectives } & \ldots & \ldots & \ldots & \ldots & \ldots & \ldots & \ldots & \ldots & \ldots & 3\end{array}$

$\begin{array}{lllllllllll}\text { Chapter 2: } & \text { Literature review } & \ldots & \ldots & \ldots & \ldots & \ldots & \ldots & \ldots & \ldots & 4\end{array}$

$\begin{array}{lllllllll}2.1 & \text { Planar flame speed vs equivalence ratio } & \ldots & \ldots & \ldots & \ldots & \ldots & 4\end{array}$

$\begin{array}{llllllll}2.2 & \text { Study of flame propagation mechanism } & \ldots & \ldots & \ldots & \ldots & \ldots & 5\end{array}$

2.3 Study of particle-gaseous premixed flames
$\ldots$

$\begin{array}{llllllllllll}\text { Chapter 3: Gaseous flame formation } & \ldots & \ldots & \ldots & \ldots & \ldots & \ldots & \ldots & 8\end{array}$

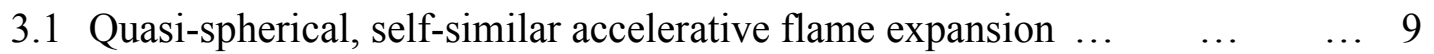

$\begin{array}{llllllllll}3.2 & \text { Finger-like flame acceleration } & \ldots & \ldots & \ldots & \ldots & \ldots & \ldots & 10\end{array}$

$\begin{array}{llllllllll}3.3 & \text { Wall friction and obstacles } & \ldots & \ldots & \ldots & \ldots & \ldots & \ldots & \ldots & 14\end{array}$

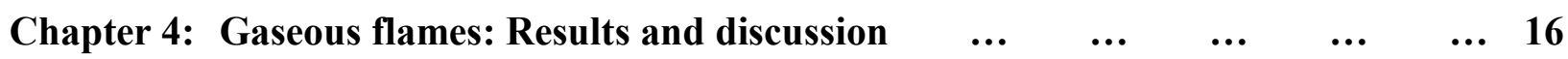

Chapter 5: Effect of combustible dust on flame propagation in tunnels $\quad \ldots \quad \ldots \quad 24$

$\begin{array}{lllllllllllll}5.1 & \text { Motivation } & \ldots & \ldots & \ldots & \ldots & \ldots & \ldots & \ldots & \ldots & \ldots & 24\end{array}$

$\begin{array}{llllllllll}5.2 & \text { Mathematical model } & \ldots & \ldots & \ldots & \ldots & \ldots & \ldots & \ldots & 25\end{array}$

$\begin{array}{lllllllll}5.2 .1 & \text { Effect of equivalence ratio promotion } \ldots & \ldots & \ldots & \ldots & \ldots & 25\end{array}$

$\begin{array}{lllllllllll}5.3 & \text { Results and discussion } & \ldots & \ldots & \ldots & \ldots & \ldots & \ldots & \ldots & 32\end{array}$

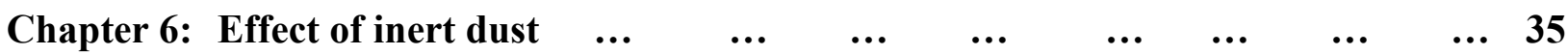

$\begin{array}{lllllllllll}6.1 & \text { Mathematical model } & \ldots & \ldots & \ldots & \ldots & \ldots & \ldots & \ldots & 35\end{array}$

$\begin{array}{llllllllll}6.2 & \text { Results and discussion } & \ldots & \ldots & \ldots & \ldots & \ldots & \ldots & \ldots & 41\end{array}$

$\begin{array}{lllllllllll}\text { Conclusion and future work } & \ldots & \ldots & \ldots & \ldots & \ldots & \ldots & \ldots & \ldots & 47\end{array}$

$\begin{array}{lllllllllllll}\text { References } & \ldots & \ldots & \ldots & \ldots & \ldots & \ldots & \ldots & \ldots & \ldots & \ldots & 49\end{array}$ 


\section{Nomenclature:}

\section{Symbols}

\begin{tabular}{|c|c|}
\hline$c$ & Concentration of particles $\left(\mathrm{kg} / \mathrm{m}^{3}\right)$ \\
\hline$c_{o}$ & Local sound speed (m/s) \\
\hline$C$ & Total heat capacity $(\mathrm{kJ} / \mathrm{kg})$ \\
\hline$C_{p}$ & Heat capacity of fresh air fuel mixture $(\mathrm{kJ} / \mathrm{kgK})$ \\
\hline$C_{s}$ & Heat capacity of dust particles $(\mathrm{kJ} / \mathrm{kgK})$ \\
\hline$H$ & Distance of the point of ignition from a tunnel wall (m) \\
\hline$K_{D L}$ & Darrieus-Landau cutoff wave number $\left(\mathrm{m}^{-1}\right)$ \\
\hline$K_{u}$ & Thermal conductivity $\left(\mathrm{K} \cdot \mathrm{m} \cdot \mathrm{W}^{-1}\right)$ \\
\hline$L e$ & Lewis number \\
\hline$L_{f}$ & Flame thickness (m) \\
\hline$M_{\text {fuel }}$ & $\begin{array}{l}\text { Total amount of fuel available per unit time per unit volume (taking into } \\
\text { account the volatilities) }\left(\mathrm{kg} / \mathrm{m}^{3} \mathrm{~s}\right)\end{array}$ \\
\hline$M_{C H 4}$ & $\begin{array}{l}\text { Total amount of methane available for combustion per unit time per unit } \\
\text { volume }\left(\mathrm{kg} / \mathrm{m}^{3} \mathrm{~s}\right)\end{array}$ \\
\hline$n$ & Number of particles per unit volume $\left(\mathrm{m}^{-3}\right)$ \\
\hline $\operatorname{Pr}$ & Prandtl number \\
\hline$Q$ & Heat released during combustion $(\mathrm{kJ})$ \\
\hline$r$ & Distance along radial direction $(\mathrm{m})$ \\
\hline$R$ & Characteristic length scale (m) \\
\hline $\operatorname{Re}$ & Flame propagation Reynolds number \\
\hline
\end{tabular}




$\begin{array}{ll}R_{f} & \text { Radius of the flame skirt (m) } \\ T & \text { Temperature (K) } \\ T_{b} & \text { Adiabatic flame temperature (K) } \\ T_{s} & \text { Surface temperature of dust particles } \\ T_{v} & \text { Temperature at which devolatilization process is initiated } \\ t_{r} & \text { The time which the coal particles stay in the flame (sec) } \\ t_{s p h} & \text { Time Taken for evolution from spherical flame to figure flame (sec) } \\ t & \text { Time taken for the flame skirt to contact the wall (sec) } \\ t_{w a l l} & \text { Unstretched laminar flame speed (m/s) } \\ S_{L} & \text { Instantaneous global flame speed with respect to the fuel mixture (taking } \\ U_{D L} & \text { into account the Darrieus-Landau instability) (m/s) } \\ Z_{r . u . d} & \left.\text { Devolatalization rate (kg/m }{ }^{3} \mathrm{~s}\right) \\ Z_{t i p} & \text { Total mass of volatilities released per unit volume } \\ w_{v}^{\prime} & \text { Distance along axial direction (m) } \\ w_{v} & \text { Zeldovich number } \\ z & \text { Flame tip position (m) }\end{array}$

Greek Symbols

kinetic viscosity $\left(\mathrm{m}^{2} / \mathrm{s}\right)$ 


$\begin{array}{ll}\phi & \text { Fresh air fuel equivalence ratio } \\ \phi_{l} & \text { Fuel lean limit equivalence ratio } \\ \phi_{r} & \text { Fuel rich limit equivalence ratio } \\ \sigma_{W} & \text { Flame acceleration rate } \\ \rho & \text { Density }\left(\mathrm{g} / \mathrm{cm}^{3}\right) \\ \lambda_{D L} & \text { Darrieus-Landau cutoff wavelength }\end{array}$

\section{Subscripts \\ Superscripts}

$b$

$f$

$S$

$r$

$z$

1

2

6

6
Burnt matter

Flame conditions

Dust particles

Radial coordinate

Axial coordinate

Fresh air fuel mixture

Burnt matter

Property changed due to coal particle

Property changed due to inert particle 


\section{List of Tables:}

Table 1. Various mechanisms of flame acceleration in tunnels/tubes/channels 2

Table 2. Propane-air flame parameters 4

Table 3. Methane-air flame parameters $\quad 4$

Table 4. Fuel dependent coefficients for laminar burning velocity correlation 4 


\section{List of Figures:}

Fig. 1: Quasi-spherical flame expansion: the stages of ignition, uniform propagation of a smooth front (a) as well as self-similar acceleration of a cellular front (b).

Fig. 2: Finger-like flame acceleration.

Fig. 4.1: Time limitations of the finger flame acceleration, $t_{s p h}$, Eq. (3.21), and $t_{\text {wall }}$ Eq. (3.32), versus the equivalence ratio $\phi$ for propane-air and methane-air flames, $R=H=1 \mathrm{~m}, \mathrm{n}=1.4$.

Fig. 4.2: Time evolution of flame tip position $Z_{t i p}$ in a stoichiometric mixture within the interval $t_{s p h}<t<t_{\text {wall }}$, with $t_{\text {sph }}$ given by Eq. (3.23), and $t_{\text {wall }}$ given by Eq. (3.29). The methane-air and propane-air mixtures are shown by the dashed and solid lines, respectively.

Fig. 4.3: Flame tip position at the time when the finger flame mechanism terminates, $Z_{\text {tip }}\left(t_{\text {wall }}\right)$, Eq. (3.31) versus the equivalence ratio $\phi$. The methane-air and propaneair mixtures are shown by the dashed and solid lines, respectively.

Fig. 4.4: Time evolution of flame tip velocity $d Z_{\text {tip }} / d t$, Eq. (3.27), within the interval $t_{\text {sph }}<t<t_{\text {wall }}$, with $t_{\text {sph }}$ of Eq. (3.21) and $t_{\text {wall }}$ of Eq. (3.32). The methane-air and propane-air mixtures are shown by the dashed and solid lines, respectively.

Fig. 4.5: The maximal flame tip velocity $\left(d Z_{\text {tip }} / d t\right)_{\text {wall }}$, Eq. (3.27), versus the equivalence ratio $\phi$. The methane-air and propane-air mixtures are shown by the dashed and solid lines, respectively.

Fig. 4.6: Flame run up distance, $Z_{\text {r.u.d }}$, Eq. (4.2) versus the equivalence ratio $\phi$. The methane-air and propane-air mixtures are shown by the dashed and solid lines, respectively. 
Fig. 4.7: Time Evolution of flame tip acceleration $d^{2} Z_{\text {tip }} / d t^{2}$, Eq. (3.28) within the interval $t_{s p h}<t<t_{\text {wall }}$ between $t_{s p h}$, Eq. (3.23), and $t_{\text {wall }}$, Eq. (3.31). The methaneair and propane-air mixtures are shown by the dashed and solid lines, respectively.

Fig. 4.8: Maximal flame tip acceleration $\left(d^{2} Z_{t i p} / d t^{2}\right)_{\text {wall }}$, Eq. (3.28), taken at the termination time $t_{\text {wall }}$, Eq. (3.29), vs the equivalence ratio $\phi$. The methane-air and propane-air mixtures are shown by the dashed and solid lines, respectively.

Fig 5.1: The modified Equivalence ratio vs the concentration of particles of size 75 28 microns.

Fig 5.2: The modified Equivalence ratio vs the concentration of particles of size 25 microns.

Fig 5.3: The modified flame temperature, Eq. (5.9), vs the dust concentration of particle size 75 microns.

Fig 5.4: The modified flame temperature, Eq. (5.9), vs the dust concentration of particle size 25 microns.

Fig 5.5: The modified burning velocity, Eq. (5.10), vs the dust concentration of particle size 75 microns for different equivalence ratios.

Fig 5.6: The modified flame temperature, Eq. (5.10), vs the dust concentration of particle size 25 microns for different equivalence ratios.

Fig 5.7: Comparison of flame speeds vs the dust concentration of particle size for fixed equivalence ratio.

Fig 5.8: Flame tip position, Eq. (5.15), at the time $t_{\text {wall }}$ given by Eq. (5.13), vs the concentration of coal particles for a set of fixed equivalence ratios. 
Fig 5.9: The time limitations of the finger flame acceleration, $t_{s p h}$ of Eq. (5.12) and $t_{\text {wall }}$ of Eq. (5.13), versus the equivalence ratio $\phi$ for methane-air mixture with and without dust particles.

Fig. 5.10: The maximal flame tip velocity $\left(d Z_{\text {tip }} / d t\right)_{\text {wall }}$, Eq. (5.16), at $t_{\text {wall }}$, Eq. (5.14), vs the equivalence ratio $\phi$ for the methane-air without particles and methaneair with particles mixtures of size 75 microns.

Fig. 5.11: Evolution of flame tip velocity $d Z_{t i p} / d t$, Eq. (5.16), vs the time interval between $t_{s p h}$, Eq. (5.13), and $t_{\text {wall }}$, Eq. (5.14), $t_{s p h}<t<t_{\text {wall }}$. The methane-air with particles and methane-air without particles mixtures are shown for the lean fuel ratio $\phi=0.7$.

Fig 6.1: Decrease in flame temperature $T_{f}^{\prime \prime}$, Eq. (6.5), vs concentration of particles $c_{s}$ with size of 75 microns.

Fig 6.2: The decrease in the flame temperature $T_{f}^{\prime \prime}$, Eq. (6.5), vs the concentration of particles $c_{s}$ for the particles of size 120 microns.

Fig 6.3: Decrease in the burning velocity $S_{L}^{\prime \prime}$, Eq. (6.7), vs concentration of particles $c_{S}$ with size of 75 microns.

Fig 6.4: Decrease in the burning velocity $S_{L}^{\prime \prime}$, Eq. (6.7), vs concentration of particles $c_{S}$ with size of 120 microns.

Fig. 6.5: The flame temperature $T_{f}^{\prime \prime}$ with the dust concentration for the particles of size 75 and 120 microns and fixed equivalence ratio $\phi=0.7$.

Fig. 6.6: The laminar flame speed $S_{L}^{\prime \prime}$ with the dust concentration for the particles of 40 size 75 and 120 microns and fixed equivalence ratio $\phi=0.7$. 
Fig 6.7: The time limitations of the finger flame acceleration, $t_{s p h}$ of Eq. (6.12), and $t_{\text {wall }}$ of Eq. (6.13), at equivalence ratio $\phi=0.7-0.9$ versus concentration of particles for methane-air flames.

Fig 6.8: The flame tip position, Eq. (6.9), at the termination time $t_{\text {wall }}$, Eq. (6.13), vs the concentration of inert particles for fixed equivalence ratio.

Fig. 6.9: The maximal flame tip velocity $\left(d Z_{\text {tip }} / d t\right)_{\text {wall }}$, Eq. (6.11), at the time $t_{\text {wall }}$ of Eq. (6.13) vs the concentration of inert particles for the methane-air-inert mixtures of different equivalence ratios $\phi=0.7,0.8$ and 0.9 with particles of size 75 microns.

Fig. 6.10: Evolution of flame tip velocity $d Z_{t i p} / d t$, Eq. (6.10), vs the time interval between $t_{s p h}$, Eq. (6.12), and $t_{\text {wall }}$, Eq. (6.13), $t_{s p h}<t<t_{\text {wall }}$. The methane-air with inert particles and methane-air without particles mixtures are shown for the lean fuel ratio $\phi=0.7$.

Fig 6.11: The time limitations of the finger flame acceleration, $t_{s p h}$ of Eq. (6.12) and 45 (5.12), and $t_{\text {wall }}$ of Eq. (6.13) and (5.13), at equivalence ratio $\phi=0.7-0.9$ versus concentration of particles for methane-air flames.

Fig. 6.12: Evolution of flame tip velocity $d Z_{t i p} / d t$, Eq. (6.10) and (5.10), vs the time interval between $t_{s p h}$, Eqs. (6.12) and (5.12), and $t_{\text {wall }}$, Eqs. (6.13) and (5.13). $t_{\text {sph }}<t<t_{\text {wall }}$. The methane-air with inert particles, combustible and without particles mixtures are shown for the lean fuel ratio $\phi=0.7$. 


\section{Chapter 1: Introduction}

\subsection{Fundamentals of combustion}

Combustion is a chemical reaction, where oxidation of a combustible fuel takes place. It is a selfsustained chemical process in which heat is released due to overall exothermic chemical reaction. Practical applications of combustion include, in particular, power plants, chemical industries, domestic burners and automobiles. Combustion can be classified according to how the fuel and oxidizer are placed before the reaction happens: premixed and non-premixed burning. The present work is restricted to premixed combustion, i.e. the mixing of the fuel and oxidizer has completed before the ignition (for comparison, in the non-premixed combustion mode, the fuel and the oxidizer would remain separated until they enter the burning zone). In fact, premixed combustion occurs in two distinctive regimes: a flame (also known as a deflagration) and a detonation. In the case of a flame, the reaction propagates due to thermal conduction, transferring energy from the hot burnt matter to the cold fuel-air premixture. In a detonation, the reaction propagates due to shock waves, which compress the air-fuel mixture to higher temperature. A nominal, planar flame propagates slowly, of the order of $1 \mathrm{~m} / \mathrm{s}$, being thereby a subsonic burning regime, whereas a detonation wave spreads with a speed of the order of $\sim 1000 \mathrm{~m} / \mathrm{s}$, being a supersonic burning regime. However, a sporadic deflagration-to-detonation (DDT) transition may occur, being a powerful effect, especially in those scenarios where it has not been predicted or controlled yet (say, accidental explosions in coal mines). One of the purposes of the present study is to develop a predictive scenario of DDT due to accidental fire explosions in coal mines and provide a strategy to mitigate the process. 


\subsection{Mining Fire and Explosion}

Mining industry has ones of the highest injury and fatality rates. Although various mining accidents are caused by many reasons, spontaneous methane explosions in dusty coal mines, followed by comprehensive methane-air-dust fires, constitute the most typical hazard. The primary outcome of the present work is a quantitative predictive scenario of such fires and explosions. One of the key characteristics of the fire spreading is the unstretched laminar flame speed $S_{L}$, which is a function of thermal-chemical properties of the fuel mixture. In particular, $S_{L}$ depends on the premixture equivalence ratio $\phi$. For dusty-gaseous environment, $S_{L}$ also depends on the dust parameters such as the dust particle size and the dust concentration. At the same time, a fire front, as well as any practical flame front, is not planar, but strongly corrugated; thereby, it consumes more fuel per unit time and propagates faster. Typical distinctive scenarios of flame corrugation/acceleration are summarized in Table 1. In this work, we employ them to quantify the mining fire scenario and fix a relevance of any particular mechanism to the mining passage.

\begin{tabular}{|l|l|l|l|}
\hline $\begin{array}{l}\text { Origin of flame } \\
\text { corrugation/acceleration }\end{array}$ & $\begin{array}{l}\text { Re- } \\
\text { dependence }\end{array}$ & $\begin{array}{l}\text { Relevance } \\
\text { mines }\end{array}$ & $\begin{array}{l}\text { Relevance to } \\
\text { micro-scales }\end{array}$ \\
\hline Flame Instability & YES, $\uparrow$ Re & YES & NO \\
\hline Finger Flame & NO & YES & YES \\
\hline Wall Friction & YES, $\downarrow$ Re & NO & YES \\
\hline Obstacles & NO & It depends & It depends \\
\hline Turbulence & YES & YES & It depends \\
\hline
\end{tabular}

\section{Table 1. Various mechanisms of flame acceleration in tunnels/tubes/channels}

The Reynolds number related to flame propagation is defined as

$$
\operatorname{Re}=\frac{S_{L} R}{v}=\frac{R}{\operatorname{Pr} L_{f}}
$$

where $\operatorname{Pr}$ and $L_{f}$ are the Prandtl number and the flame thickness, respectively. Also the flame 
thickness is defined as $D_{t h} / S_{L}$, where $D_{t h}$ is thermal diffusivity. First of all, obviously, the Reynolds number increases with increasing radius of the tube/channel. In this regard, the combustion instability developing on a flame front depends on the tube radius, and the effect of the instability increases with the increasing radius, whereas when confined to micro scales there is no scope for the flame instability to develop. As a result the flame instability mechanism is relevant to mining fire scenario, however it is not relevant to micro-scale combustion. Secondly, the finger flame scenario, which is independent of the Reynolds number, has the same effect in both micro and macro scales. The remaining mechanisms are described in Chapter 4.

\subsection{Objectives}

The major objective of this work is to develop a predictive scenario of a mining fire, aiming to provide the guidance for preventing and controlling gas explosion disasters in mines. This work primarily looks into the ways of suppressing terminating a spreading fire and/or prevention of its initiation or - if it is not possible to terminate a fire - at least mitigate its propagation and consequences. Eventually, if DDT scenario is unavoidable, then it is aimed to investigate it and find the ways to mitigate the detonation hazards. Overall, in terms of predicting the risks of fire scenarios, this work is split into three objectives:

1. To predict the timing and locus for each stage of the flame evolution such as transition from a spherical shape to a finger-like shape and that when a flame skirt contacts a passage wall.

2. To predict the speed of flame spreading and run up distance before the DDT event occur.

3. To extend the formulation to dusty-gaseous environment in coal mines. 


\section{Literature Review}

\subsection{Planar Flame Speed vs Equivalence ratio}

We start with homogeneously gaseous methane-air and propane-air flames, of various equivalence ratios, $\phi$, and then extend the analysis to the situation of methane-air-dust environment. The thermal expansion $\Theta$, which changes with respect to the equivalence ratio $\phi$ is the fresh fuel to the burnt matter density ratio. The planar flame speed $S_{L}$ is presented versus $\phi$ in Table 2, for propane-air premixture, and Table 3, for methane-air premixture.

\begin{tabular}{|c|c|c|c|c|c|c|c|c|c|}
\hline$\phi$ & 0.63 & 0.7 & 0.8 & 0.9 & 1.0 & 1.1 & 1.2 & 1.3 & 1.4 \\
\hline$\Theta$ & 6.04 & 6.56 & 7.15 & 7.66 & 8.02 & 8.08 & 8.0 & 7.88 & 7.74 \\
\hline$S_{L}(m / s)$ & 0.147 & 0.217 & 0.303 & 0.374 & 0.418 & 0.429 & 0.399 & 0.322 & 0.226 \\
\hline
\end{tabular}

Table 2. Propane - Air Flame Parameters [1]

\begin{tabular}{|c|c|c|c|c|c|c|c|c|c|}
\hline$\phi$ & 0.6 & 0.7 & 0.8 & 0.9 & 1.0 & 1.1 & 1.2 & 1.3 & 1.4 \\
\hline$\Theta$ & 5.54 & 6.11 & 6.65 & 7.12 & 7.48 & 7.55 & 7.43 & 7.28 & 7.09 \\
\hline$S_{L}(m / s)$ & 0.088 & 0.203 & 0.286 & 0.336 & 0.353 & 0.342 & 0.306 & 0.249 & 0.179 \\
\hline
\end{tabular}

Table 3. Methane - Air Flame Parameters [1]

\begin{tabular}{|c|c|c|c|c|}
\hline fuel & $S_{L, \max }(m / s)$ & $\phi_{\max }$ & $\phi_{l}$ & $\phi_{r}$ \\
\hline methane & 0.35 & 1.06 & 0.533 & 1.68 \\
\hline propane & 0.41 & 1.08 & 0.536 & 2.50 \\
\hline
\end{tabular}

Table 4. Fuel dependent coefficients for laminar burning velocity correlation [2]

The dependence of $S_{L}=S_{L}(\phi)$, can also be approximated as [2]

$$
\begin{aligned}
& S_{L}(\phi)=S_{L, \max }\left(\frac{\phi-\phi_{l}}{\phi_{\max }-\phi_{l}}\right)^{a}\left(\frac{\phi_{r}-\phi}{\phi_{r}-\phi_{\max }}\right)^{b}, \\
& a=2\left(\frac{\phi_{\max }-\phi_{l}}{\phi_{\max }-\phi_{l}}\right), \quad b=2\left(\frac{\phi_{r}-\phi_{\max }}{\phi_{r}-\phi_{l}}\right),
\end{aligned}
$$


where $\phi_{l}$ is fuel-lean limit, $\phi_{r}$ is fuel-rich limit, and $\phi_{\max }$ corresponds to equivalence ratio for maximum laminar burning velocity $S_{L, \max }$. For the calculation of Eq. (2.1), related parameters can be seen in Table 4 .

\subsection{Study on flame propagation mechanism}

To prevent gas explosions, researchers used to carry out exhaustive explorations of the explosive limits and identify the characteristic detonation dynamics and influencing factors [3-5]. The studies showed that the equivalence ratio is one of primary parameter influencing the processes of combustion and explosion. Specifically, it influences, crucially, the flame propagation and acceleration mechanisms as well as the flame structure and stability limits [6]. A flame propagates as a curved front rather than maintaining a stable planar front, in particular, because of the combustion instabilities [7]. Clanet and Searby [8] first explained the acceleration mechanism at the early stages of burning in tubes, and the analytical formulation was recently developed by Bychkov et al [9]. According to [8,9], the acceleration happens due to the initial ignition geometry in the tube axis when a flame develops into a finger-shaped front, with the surface area and the flame tip velocity growing exponentially in time. It is noted that the flame accelerates quite fast, but only for a short time, until the flame skirt contacts the wall. The analytical formulas for the acceleration rate, for the flame shape and for the velocity profile in the flow pushed by the flame were developed.

A flame in a tube or channel can spontaneously accelerate until it triggers a detonation [10-15], which states one of the most important and, also, one of the complicated problems in combustion science. Various experimental studies $[16,17]$ have shown the following steps in the DDT a flame accelerates, pushes compression waves and weak shocks, which interact, get stronger, compress 
and heat the fresh fuel mixture, which finally explodes somewhere between the leading shock and the flame front and evolves into the detonation.

As the non-uniform velocity distribution makes the flame shape curved, which increases the burning rate and drives the acceleration. The quantitative theory and modeling of wall friction (Schelkin) mechanism [18] was first studied by Bychkov et al. [19]. They considered laminar premixed flames with a realistically strong density drop at the front. Subsequently, an analytical theory of accelerating flames has also been developed by Akkerman et al. [20] for a cylindrical tube geometry with one end closed and nonslip at the walls.

In Ref. [21], it was demonstrated that how initial turbulence enhances the combustion reaction rate and heat and mass transfer efficiency, thereby enlarging the range of the explosive concentration limit. Fundamental and thorough investigation of the equivalence ratios effect on the flame microstructure and propagation behavior has not been performed yet, even though some studies regarding hydrogen fires have been recently conducted [22]. Studies of premixed flames in a compartment are required to understand the effect of the equivalence ratio on the flame propagation behavior and improve the safety of the burning process.

In the present thesis we demonstrate the inner mechanism of gas explosion, and reveal the relationship between different equivalence ratios and gas explosion dynamics, which aim to provide theoretical and experimental guidance for preventing and controlling gas explosion disasters.

\subsection{Analysis of Dust-Gas-Air Premixed Flames}

Combustion of clouds of fuel particles in a gaseous atmosphere is having importance for understanding coal dust explosions in mines. A theory of flame propagation in a uniform cloud of 
particles was developed long ago [23-25]. The flame propagation model has been developed by Refs. [26-27] depends on the number of density and initial size of fuel particles. Other important theoretical study of the structure of premixed flames, containing uniformly distributed volatile fuel particles, in an oxidizing gas mixture is analyzed by Seshadri [28]. It is presumed that the fuel particles vaporize first to yield a gaseous fuel, which is subsequently oxidized in the gas phase. This process is resulted with temperature increase in the reaction zone which promotes the equivalence ratio and thereby velocity. 


\section{Chapter 3: Gaseous Flame Formulation}

We start with a premixed fire spreading in a homogenously-gaseous environment. The effect of multi-phase impurities such as combustible and/or inert dust will be considered later, in Chapters 5 and 6. To be specific, we consider an accidental ignition of a methane-air (or propane-air) premixture occurring at a distance $H$ from a tunnel wall, as illustrated in Fig. 1. A flame propagates outwardly from the ignition point. The flame front is initially spherically-smooth, because any tendency to develop a combustion instability is suppressed by the stretch effect, which is induced by an expanding flame. The process is controlled by the interplay between the flame stretch and the mixture (non) equi-diffusion. The critical issue at an early stage of a fire is whether an embryonic flame can sustain. In this respect, the front evolution depends on the thermal to molecular diffusivity ratio, i.e. the Lewis number, $L e$. Indeed, the sustained flame propagation is possible for $L e>1$ mixtures, whereas for $L e<1$ combustibles, a flame embryo needs to attain a minimum radius, through the initial spark energy, before sustained propagation is possible [29]. Consequently, keeping $L e<1$ in the mining environment may improve the fire safety standards.

(a)

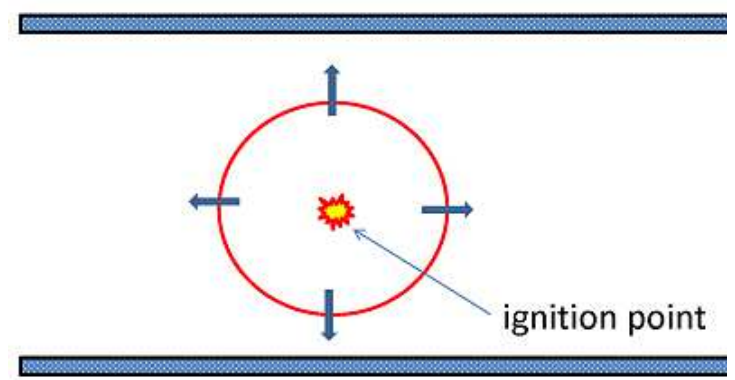

Fig. 1: Quasi-spherical flame expansion: the stages of ignition, uniform propagation of a smooth front (a) as well as self-similar acceleration of a cellular front (b). (b)

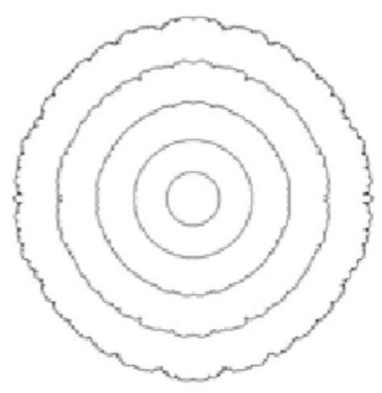




\subsection{Stage 1: Quasi-Spherical, Self-Similar Accelerative Flame Expansion}

Assume, nevertheless, that an embryonic flame has survived and keeps propagating. In the present study, we are interested, mostly, in large-scale fires; hence, the flame stretch can be neglected. Then, at the early stage of burning, the flame front expands with a constant speed with respect to the ignition point, $d R_{f} / d t=\Theta S_{L}$, where $R_{f}$ is the flame radius. As the flame "ball" grows in size and the stretch intensity diminishes, the diffusional-thermal cells would develop over the surface of $L e<1$ flames. Subsequently, the flame thickness relative to the global flame radius is reduced, leading to the onset of the hydrodynamic (Darrieus-Landau; DL) instability mode. The latter generates hydrodynamic cells over the flame surface, regardless of $L e$, and it will eventually control the surface morphology. The continuous generation of new cells leads to the continuous increase in the flame surface density and thereby an expanding flame self-accelerates in a scaleinvariant (self-similar) manner; see Fig. 1b. According to numerous experimental studies, a reasonable fitting law for such acceleration is [30]

$$
R_{f}=R_{0}+C t^{n} \approx C t^{n},
$$

where $R_{0}$ plays the role of a critical radius related to the transition to the cellular flame structure (it can be neglected within the frame of the large-scale formulation); the exponent $n \approx 1.3-1.4$ in the most of studies; and the factor $C$ can be evaluated as [30]

$$
C=k_{D L}^{n-1}\left(\Theta S_{L} / n\right)^{n},
$$

where $k_{D L}^{n-1}$ is a DL cutoff wavenumber that appears in the Pelce-Clavin dispersion relation [31]

$$
\sigma_{D L}(k)=\Gamma(\Theta) S_{L} k\left(1-\frac{k}{k_{D L}}\right), \quad \Gamma(\Theta)=\frac{\Theta}{\Theta+1}\left[\left(\Theta+1-\frac{1}{\Theta}\right)^{1 / 2}-1\right] .
$$


The quantity $k_{D L}$ is coupled to the DL critical wavelength, $k_{D L}=2 \pi / \lambda_{D L}$, where $\lambda_{D L}$ is the critical wavelength, which in turn depends on thermal expansion $\Theta$ and is proportional to the flame thickness, such that $\lambda_{D L} \approx(30 \sim 100) L_{f}$ for $\Theta=5 \sim 8$. In this work, we employ the following formula for the DL cutoff [31]

$$
\lambda_{D L}=2 \pi L_{f}\left(1+\frac{(\Theta+1)}{(\Theta-1)^{2}} \Theta \ln \Theta\right)
$$

With the power-law flame acceleration, Eq. (3.1), the global (radial) flame speed with respect to the ignition point is not a constant $\Theta S_{L}$ anymore, but a time-dependent quantity

$$
d R_{f} / d t=n C t^{n-1}=\left(k_{D L} / n\right)^{n-1}\left(\Theta S_{L}\right)^{n} t^{n-1} .
$$

To evaluate the instantaneous global flame speed with respect to the fuel mixture we divide Eq. (3.5) by $\Theta$, with the result

$$
U_{D L}=\frac{1}{\Theta} \frac{d R_{f}}{d t}=\frac{n C}{\Theta} t^{n-1}=S_{L}^{n}\left(\frac{\Theta}{n} k_{D L}\right)^{n-1} t^{n-1} .
$$

\subsection{Stage 2: Finger-like Flame Acceleration}

Generally speaking, Eqs. (3.1) - (3.6) describe the accelerative flame expansion in an opening. However, in the practical reality, the shape of a flame front approaching (even not contacting!) the tunnel wall gets modified, forming two outwardly propagating "finger-like" fronts, as illustrated in Fig. 2. Expansion of the burning matter leads to a strong flow in the axial direction, which drifts the tip of a "finger"-shaped flame. Because of the elongated shape, the surface area of the flame front is much larger than the passage cross section. As a result, the flame accelerates. However, this, second stage of acceleration terminates as soon as the flame skirt contacts the passage sidewall. By the end of this process, the distance from the ignition point to the flame tip position 
is considerably larger than the passage radius. We next combine the above analyses of an expanding flame with a finger-flame formulation [9]. Specifically, we reconcile the study [9], with a time-dependent quantity $U_{D L}(t)$, Eq. (3.6), playing the role of $S_{L}$. A rational for this consideration is the scale- and time separation between the formulations: the tunnel width is much larger than the DL cells, and the finger-flame acceleration is much stronger than the DL one.

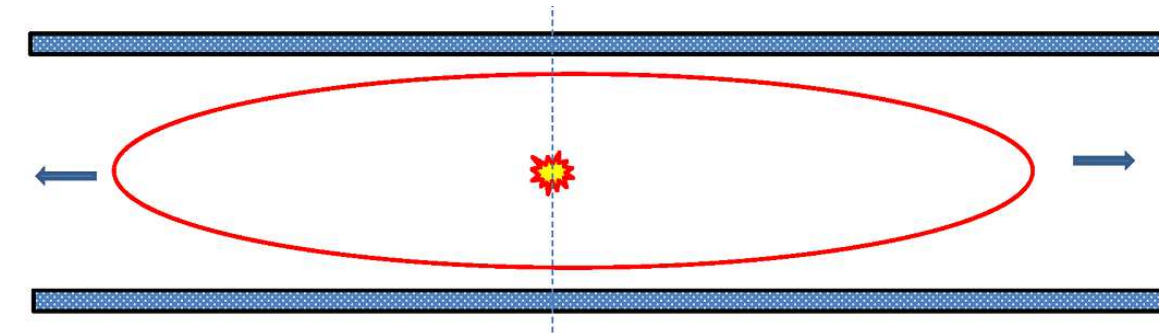

Fig. 2: Finger-like flame acceleration.

As the flow is separated into two regions, namely, the fresh fuel mixture and the burnt matter, the continuity equation for an incompressible flow in the axisymmetric cylindrical coordinates reads

$$
\frac{1}{r} \frac{\partial\left(r u_{r}\right)}{\partial r}+\frac{\partial u_{z}}{\partial z}=0 .
$$

Hereafter, we denote the axial and radial flow velocities ahead and behind the flame front (i.e. in the fresh fuel mixture and the burnt matter) by subscripts "1" and "2", respectively. The axial flow boundary condition at walls for fresh fuel mixture is

$$
\left.u_{z, 1}\right|_{z=0}=0 .
$$

First, we are interested in the flow along the walls for $z \rightarrow 0$. In the fresh fuel mixture for the potential flow, we look for the velocity profile in the form

$$
u_{z, 1}=A_{1}(t) z
$$

where the factor $A_{1}$ is a function of time but not space, similarly to the finger flame formulation [9]. The radial velocity in the fuel mixture is calculated from the continuity equation (3.7) as 


$$
u_{r, 1}=\frac{A_{1}(t)}{2}\left(\frac{H^{2}}{r}-r\right)
$$

We next employ the radial flow boundary conditions at the centerline and sidewall of the tunnel

$$
\left.u_{r}\right|_{r=0}=\left.u_{r}\right|_{r=H}=0 \text {. }
$$

Then the velocity distribution in the burnt matter (subscript 2) takes the form

$$
\begin{aligned}
& u_{z, 2}=A_{2}(t) z, \\
& u_{r, 2}=-\frac{A_{2}(t)}{2} r .
\end{aligned}
$$

To close the system, we consider the matching conditions at the front, $r=R_{f}$, being

$$
\begin{aligned}
& \frac{d R_{f}}{d t}-u_{r .1}=U_{D L}(t), \\
& u_{r, 1}-u_{r, 2}=(\Theta-1) U_{D L}(t), \\
& u_{z, 1}=u_{z, 2} .
\end{aligned}
$$

Here Eq. (3.14) shows that the global radial flame speed is a time-dependent quantity, which is equal to the flow velocity plus global flame speed with respect to the fuel mixture, Eq. (3.6). Equations (3.15) and (3.16) describe the jump of the normal velocity and continuity of tangential velocity at the front. Note that Eq. (3.16) works only when the flame skirt is close to the tunnel sidewall. Substituting Eqs. (3.9) - (3.13) into Eqs. (3.14) - (3.16), we find

$$
A_{1}(t)=A_{2}(t)=2(\Theta-1) U_{D L}(t) R_{f} / H^{2} .
$$

Then the evolution equation for the flame skirt reads

$$
\frac{d R_{f}}{d t}=\left\{\Theta-(\Theta-1) \frac{R_{f}^{2}}{H^{2}}\right\} U_{D L}=\left\{\Theta-(\Theta-1) \frac{R_{f}^{2}}{H^{2}}\right\} S_{L}^{n}\left(\frac{\Theta}{n} k_{D L}\right)^{n-1} t^{n-1} .
$$

Integrating Eq. (3.18) with the initial condition $\left.R_{f}\right|_{t=0}=0$, we obtain

$$
t\left(R_{f}\right)=\left\{\frac{\Theta H}{2 \alpha C} \ln \left(\frac{\Theta+\alpha\left(R_{f} / H\right)}{\Theta-\alpha\left(R_{f} / H\right)}\right)\right\}^{1 / n}=\frac{n}{\Theta S_{L}}\left\{\frac{\Theta H}{2 \alpha k_{D L}^{n-1}} \ln \left(\frac{\Theta+\alpha\left(R_{f} / H\right)}{\Theta-\alpha\left(R_{f} / H\right)}\right)\right\}^{1 / n}
$$


or

$$
\frac{R_{f}(t)}{H}=\frac{\Theta}{\alpha} \tanh \left(\frac{\alpha}{\Theta} \frac{C t^{n}}{H}\right)=\frac{\Theta}{\alpha} \tanh \left(\frac{\alpha}{\Theta H} k_{D L}^{n-1}\left(\frac{\Theta S_{L}}{n}\right)^{n} t^{n}\right),
$$

where $\alpha=\sqrt{\Theta(\Theta-1)}$. A characteristic time instant devoted to the transition from a globallyspherical to a finger-like flame shape, $t_{s p h}$, can be evaluated as

$$
t_{s p h} \approx\left(\frac{\Theta H}{2 \alpha C}\right)^{1 / n}=\frac{n}{\Theta S_{L}}\left(\frac{\Theta H}{2 \alpha k_{D L}^{n-1}}\right)^{1 / n}
$$

with the respective flame skirt location being

$$
R_{f}\left(t_{s p h}\right)=\frac{\Theta H}{\alpha} \tanh (0.5) \approx 0.46 \sqrt{\frac{\Theta}{\Theta-1}} H
$$

Substitution Eq. (3.22) into Eq. (3.6) provides the instantaneous radial flame propagation speed in the laboratory reference frame

$$
U_{D L}\left(t_{s p h}\right)=\frac{n C}{\Theta} t_{s p h}^{n-1}=n\left(\frac{C}{\Theta}\right)^{1 / n}\left(\frac{H}{2 \alpha}\right)^{(n-1) / n} .
$$

Similar to Eq. (3.14), the evolution equation for the flame tip position $Z_{\text {tip }}$ (located at a centerline, $r=0$ ) takes the form

$$
\frac{d Z_{t i p}}{d t}-u_{z, 2}=\Theta U_{D L}(t)
$$

Together with Eqs. (3.12) and (3.17), Eq. (3.24) becomes

$$
\frac{d Z_{t i p}}{d t}=2(\Theta-1) U_{D L}(t) \frac{R_{f}(t) Z_{t i p}}{H^{2}}+\Theta U_{D L}(t),
$$

with the solution

$$
Z_{\text {tip }}=\frac{\Theta H}{2 \alpha} \sinh \left(2 \frac{\alpha}{\Theta} \frac{C t^{n}}{H}\right)=\frac{H}{2} \sqrt{\frac{\Theta}{\Theta-1}} \sinh \left(2 \sqrt{\frac{\Theta-1}{\Theta}} \frac{k_{D L}^{n-1}}{H}\left(\frac{\Theta S_{L}}{n}\right)^{n} t^{n}\right) .
$$

Differentiating Eq. (3.26), we find the flame tip velocity and acceleration in the form

$$
\frac{d Z_{t i p}}{d t}=n C t^{n-1} \cosh \left(2 \frac{\alpha C t^{n}}{\Theta H}\right),
$$




$$
\frac{d^{2} Z_{t i p}}{d t^{2}}=n C t^{n-2}\left(2 \frac{\alpha C}{\Theta H} n t^{n} \sinh \left(2 \frac{\alpha C t^{n}}{\Theta H}\right)+(n-1) \cosh \left(2 \frac{\alpha C t^{n}}{\Theta H}\right)\right) .
$$

The characteristic timing when the flame skirt touches the wall (and thereby terminates the finger flame scenario) can be obtained from Eq. (3.20) with the boundary condition $R_{f}=H$, namely

$$
t_{\text {wall }, 1}=\left\{\frac{\Theta H}{2 \alpha C} \ln \left(\frac{\Theta+\alpha}{\Theta-\alpha}\right)\right\}^{1 / n}=\frac{n}{\Theta S_{L}}\left\{\frac{\Theta H}{2 \alpha k_{D L}^{n-1}} \ln \left(\frac{\Theta+\alpha}{\Theta-\alpha}\right)\right\}^{1 / n} .
$$

The second flame wing contacts the opposite wall a little latter, when $R_{f}=2 R-H$,

$$
t_{\text {wall }, 2}=\left\{\frac{\Theta(2 R-H)}{2 \alpha C} \ln \left(\frac{\Theta+\alpha}{\Theta-\alpha}\right)\right\}^{1 / n}=\frac{n}{\Theta S_{L}}\left\{\frac{\Theta(2 R-H)}{2 \alpha k_{D L}^{n-1}} \ln \left(\frac{\Theta+\alpha}{\Theta-\alpha}\right)\right\}^{1 / n} .
$$

Further simplification of Eq. (3.29) by substituting Eq. (3.31) yields

$$
\left.Z_{\text {tip }}\right|_{\text {wall }}=\Theta H
$$

For simplicity, hereafter it is assumed $H \approx R$, where $R$ is the channel radius. Then $t_{\text {wall }, 1}=t_{\text {wall }, 2}$,

$$
t_{\text {wall }}=t_{\text {wall }, 1,2}=\frac{n}{\Theta S_{L}}\left\{\frac{\Theta H}{2 \alpha k_{D L}^{n-1}} \ln \left(\frac{\Theta+\alpha}{\Theta-\alpha}\right)\right\}^{1 / n}=\frac{n}{\Theta S_{L}}\left\{\frac{\Theta R}{2 \alpha k_{D L}^{n-1}} \ln \left(\frac{\Theta+\alpha}{\Theta-\alpha}\right)\right\}^{1 / n} .
$$

\subsection{Potential Effect of Wall Friction and In-built Obstacles}

When the flame skirt contacts the tunnel sidewall, the finger-like acceleration terminates, and the flame propagation will be controlled by the so-called Schelkin mechanism [18], associated with wall friction at non-slip walls. The idea behind the Schelkin acceleration scenario is the following. A combustion process results in the expansion of the burning matter, which drives the flow of fresh fuel mixture. But due to the friction at a tube/tunnel walls, the flow becomes non uniform such that the burning matter bends the flame front, which increases the flame velocity. Therefore, the flame front accelerates. The total burning rate is given by

$$
U_{w} \propto \exp \left(\sigma_{W} S_{L} t / R\right)
$$


with the following acceleration rate

$$
\sigma_{W}=\sigma_{W}(\mathrm{Re}) \rightarrow \Theta^{2} / \mathrm{Re} .
$$

Since the time required for the flame to double its speed with the acceleration (3.34) is around 1000 seconds and also the acceleration generated by wall friction weakens with the increase in the tunnel width, as in the mines, where the diameter of a tunnel is of more than a human height, $2 \mathrm{~m}$, the Schelkin flame acceleration rate appears several orders of magnitude lower than that for Reynolds-independent finger flame acceleration. Consequently, being a key phenomenon in micro- and mesa-scale pipes, the wall friction acceleration mechanism appears not relevant in the configuration of a mining tunnel.

Overall, the expansion of hot burnt gases behind the flame front push the flow of fresh mixture according to various scenarios clarified above such as corrugation/acceleration of sphericallyexpanding flames, the finger-like flame acceleration and the wall friction mechanism. All these scenarios contribute to the enhancement of the flame propagation speed in a significant manner. One more mechanism is that associated with obstacles that a flame front meets on the way of its spreading. Obviously, the outcome of flame-obstacle interactions depends on a particular configuration and shape of such obstructions. Specifically, a "tooth-brush" array of obstacles should be mentioned as a geometry providing extremely fast acceleration and deflagration-todetonation transition [33-34]. This tooth-brush acceleration mechanism is Re-independent, being thereby applicable to both micro- and macro-scales. However, this mechanism is relevant when obstacles occupy, say, $10-90 \%$ of the flow path, which is not the case for a mining passage. Consequently, neither wall friction nor obstacles play a substantial role in a coal mine, and thereby can be omitted in this work. 


\section{Gaseous Flames: Results and Discussion}

In this Chapter we adopt the analytical formulation of Chapter 3 for a set of parameters from the practical reality, such as those from a realistic accidental explosion in a mining passage. Figure 4.1 shows the characteristic timings of the process, $t_{s p h}$, Eq. (3.21), and $t_{\text {wall }}$ Eq. (3.29), for $R=H=1 \mathrm{~m}$ and the DL exponent $n=1.4$. These quantities, $t_{s p h}$ and $t_{\text {wall }}$, play the key roles for the flame acceleration (and, thereby, fire evolution) scenario. Indeed, it is recalled that just after an ignition, the flame tip moves in the same manner as the flame skirt, Eqs. (3.14) - (3.16), exhibiting a globally-spherical (cellular) shape of an expanding flame front as soon as $t<t_{s p h}$. In contrast, when $t=t_{s p h}$, the flame skirt slows down, while the flame tip accelerates, thereby leading to a very strong flame elongation/acceleration within the time interval $t_{s p h}<t<t_{\text {wall }}$. It is recalled that this strong acceleration is limited in time: it stops wen the flame skirt touches the wall, $t=t_{\text {wall }}$

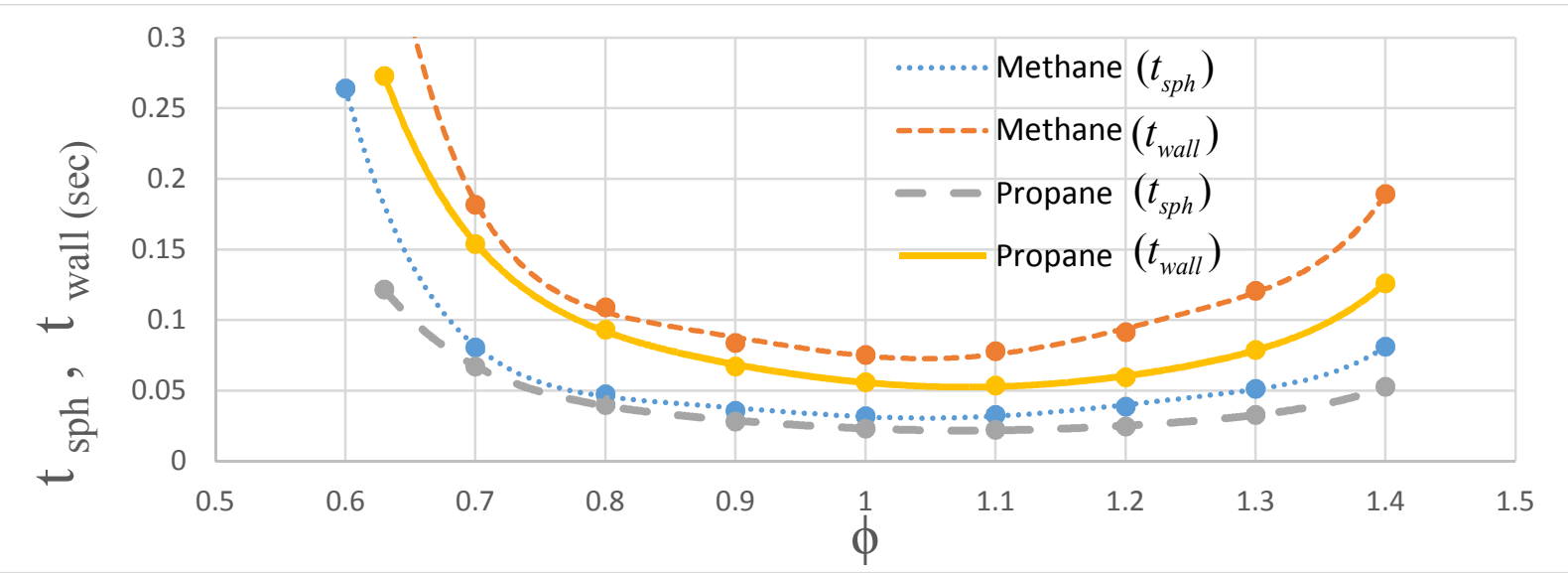

Fig. 4.1: Time limitations of the finger flame acceleration, $t_{s p h}$, Eq. (3.21), and $t_{\text {wall }}$, Eq. (3.32), versus the equivalence ratio $\phi$ for propane- and methane-air flames, $R=H=1 \mathrm{~m}, \mathrm{n}=1.4$. 


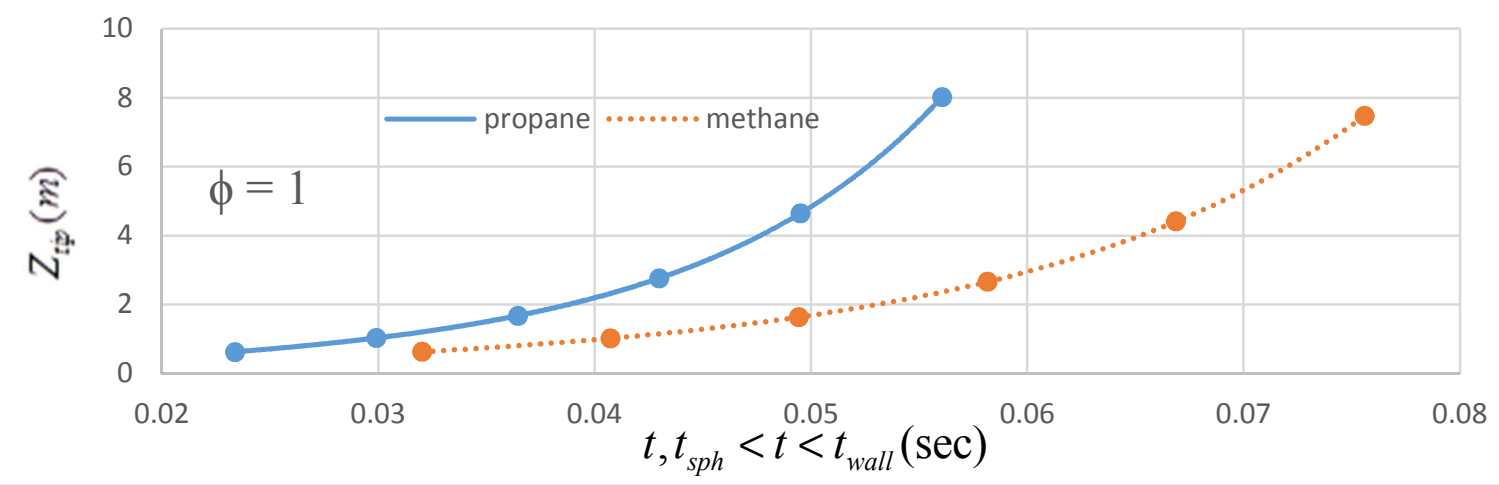

Fig. 4.2: Time evolution of flame tip position $Z_{\text {tip }}$ in a stoichiometric mixture within the interval $t_{s p h}<t<t_{\text {wall }}$, with $t_{s p h}$ given by Eq. (3.23), and $t_{\text {wall }}$ given by Eq. (3.29). The methane-air and propane-air mixtures are shown by the dashed and solid lines, respectively.

In Fig. 4.2, the flame tip position, Eq. (3.26), is plotted for stoichiometric $(\phi=1)$ methane-air and propane-air mixtures within the time interval of its finger-like acceleration, $t_{s p h}<t<t_{\text {wall }}$. It is clearly seen that the finger-like acceleration is very strong: the flame tip spreads through $8 \mathrm{~m}$ during only $\sim 0.05 \mathrm{sec}$ ! Obviously, this is crucial for miner's safety in the case of an accidental fire. We recall that the acceleration terminates at the time $t_{\text {wall }}$, when the flame skirt contacts the tunnel wall. The flame tip position at this terminal instant, $Z_{\text {tip }}\left(t_{\text {wall }}\right)$, Eq. (3.31), is presented in Fig. 4.3 versus the equivalence ratio $\phi$, within a wide range from lean to rich mixtures for both methane and propane. Obviously, $Z_{\text {tip }}\left(t_{\text {wall }}\right)$ determines a distance that a flame can travel before its skirt contacts the wall. As we can see from Eq. (3.31), this quantity is directly proportional to the thermal expansion ratio $\Theta$ and distance from the tunnel wall $H$, where the accidental ignition occurred. Again, it is noted that this value, $Z_{\text {tip }}\left(t_{\text {wall }}\right)$, is quite large, $5 \sim 8 \mathrm{~m}$, and it is maximal at stoichiometric or near-stoichiometric conditions. 


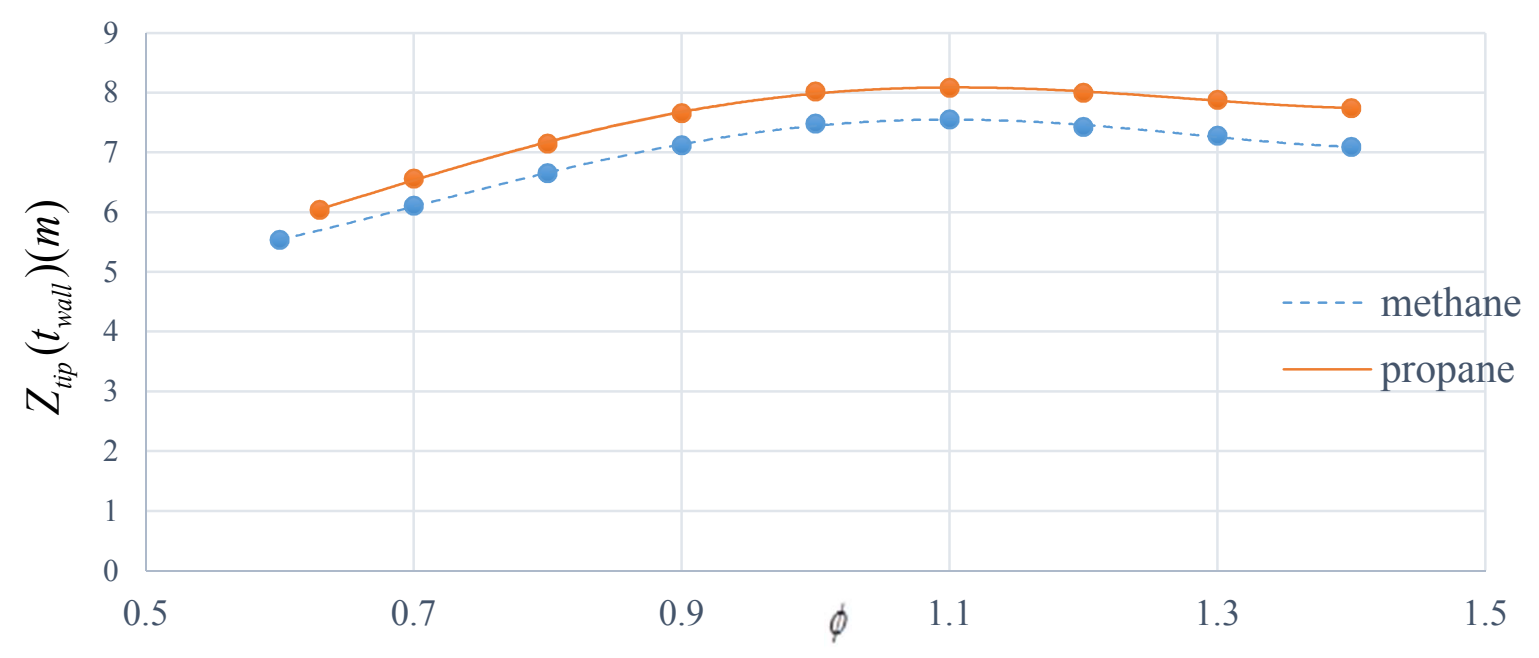

Fig. 4.3: Flame tip position at the time when the finger flame mechanism terminates, $Z_{\text {tip }}\left(t_{\text {wall }}\right)$, Eq. (3.31) versus the equivalence ratio $\phi$. The methane-air and propane-air mixtures are shown by the dashed and solid lines, respectively.

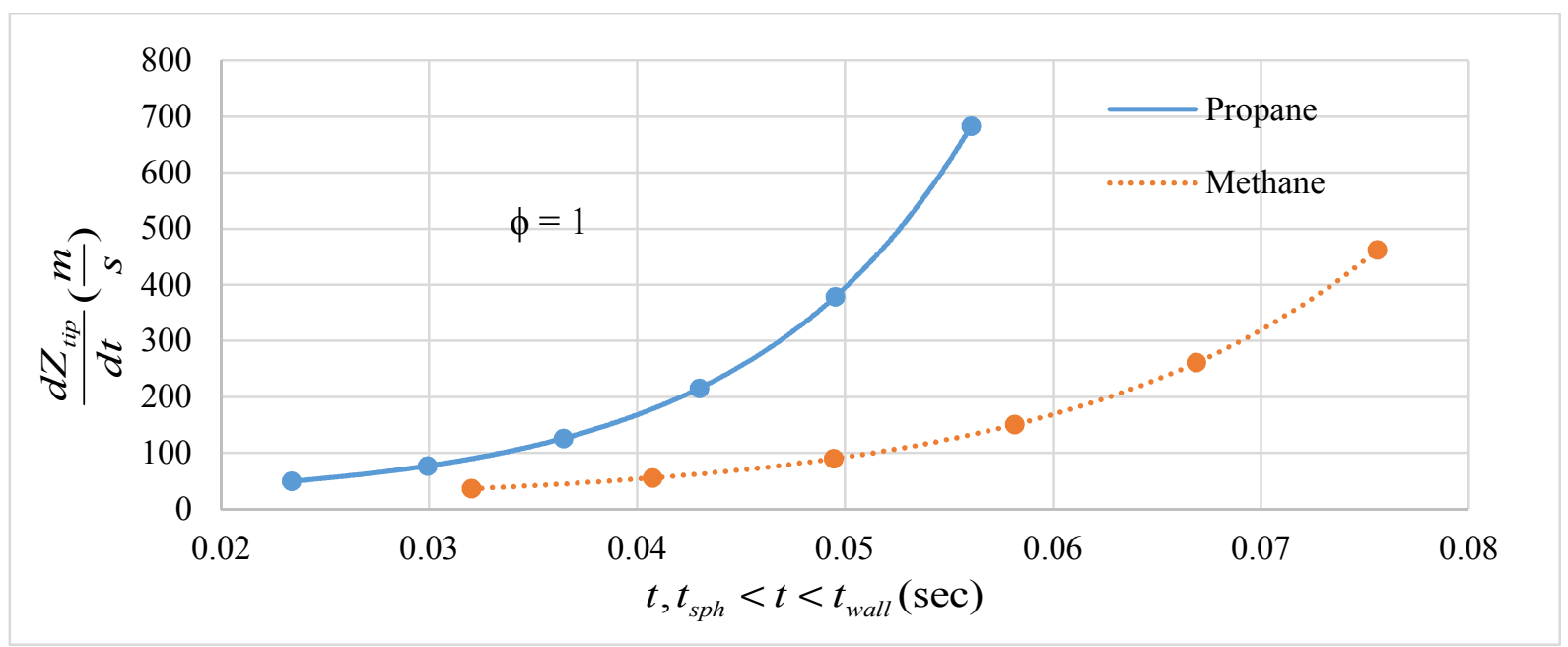

Fig. 4.4: Time evolution of flame tip velocity $d Z_{t i p} / d t$, Eq. (3.27), within the interval $t_{s p h}<t<t_{\text {wall }}$, with $t_{s p h}$ of Eq. (3.21) and $t_{\text {wall }}$ of Eq. (3.32). The methane-air and propane-air mixtures are shown by the dashed and solid lines, respectively.

Figure 4.4 presents the time evolution of the flame tip velocity, Eq. (3.27), in the time range $t_{s p h}<t<t_{\text {wall }}$, for the stoichiometric methane-air and propane-air mixtures. It is seen here that at the characteristic time $t_{s p h}$, the flame tip speed have already risen by an order of magnitude due to the Darrius-Landau instability. Then, by the time $t_{\text {wall }}$, the finger flame scenario provides one 
more order of magnitude increase in the flame velocity. As a result, the maximal flame speed is very high: $460 \mathrm{~m} / \mathrm{s}$ for a stoichiometric methane-air flame and $720 \mathrm{~m} / \mathrm{s}$ for a stoichiometric propane-air mixture, which exceeds the sound speed of the fuel mixture in both cases. The sound speeds of the mixtures are calculated using GASEQ equilibrium solver [38] for each equivalence ratio value. This solver allows us to predict the effects of changes in temperature, pressure, and concentration on a system at equilibrium. It states that if a system at equilibrium experiences a change, then the system will shift its equilibrium to try to compensate for the change.

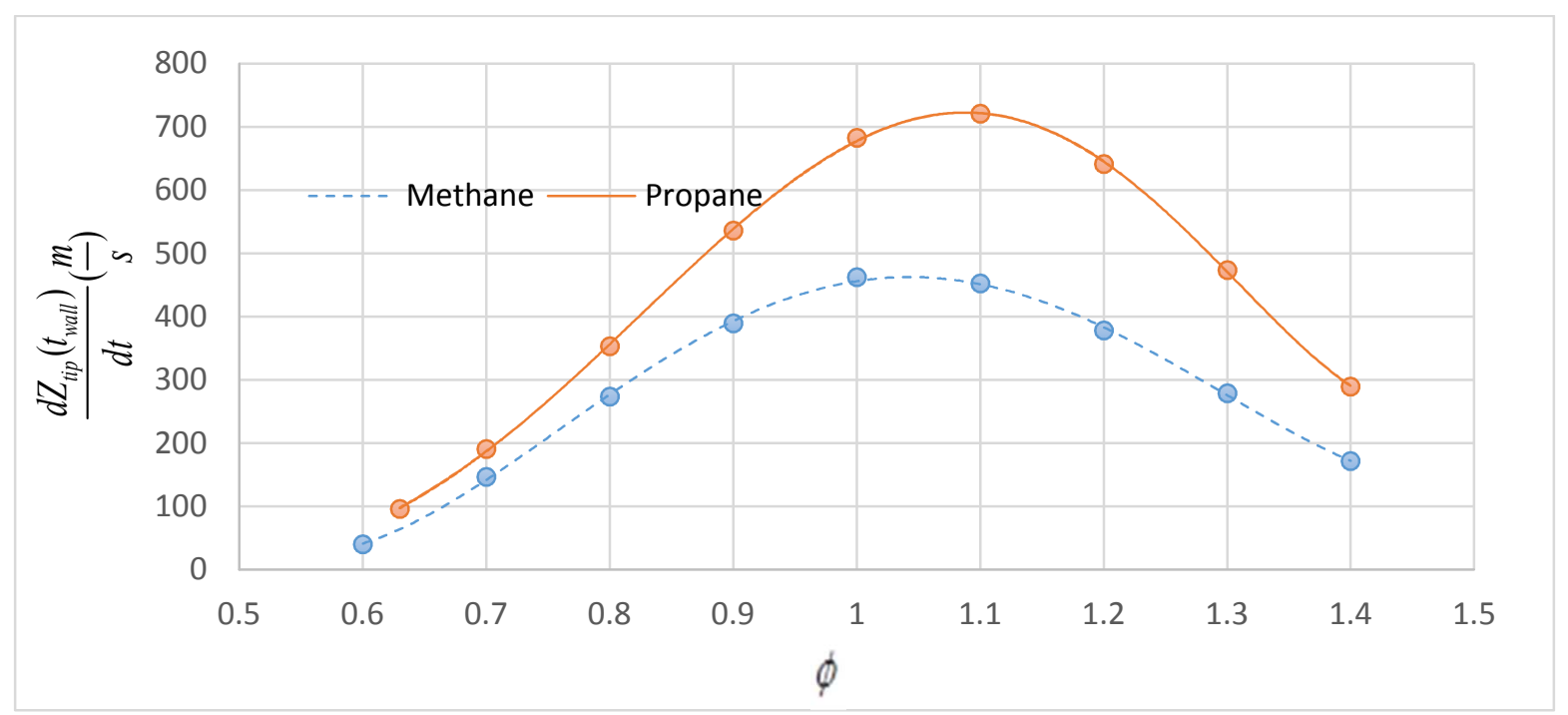

Fig. 4.5: The maximal flame tip velocity $\left(d Z_{\text {tip }} / d t\right)_{\text {wall }}$, Eq. (3.27), vs the equivalence ratio $\phi$. The methane-air and propane-air mixtures are shown by the dashed and solid lines, respectively.

Well, these quantities would nevertheless diminish if we deal with a lean or a rich premixture. This is justified in Fig. 4.5, where the maximal flame tip velocity, $\left(d Z_{\text {tip }} / d t\right)_{\text {wall }}$, Eq. (3.27), is plotted versus the equivalence ratio in the range $0.6<\phi<1.4$. We observe that the flame velocity decreases twice for $\phi=1.4$ as compared to that for $\phi=1$, while for $\phi=0.6$ such a reduction is by an order of magnitude as compared to that for $\phi=1$. 
It is seen that the flame tip velocity exceeds the associated sound speed for the propane-air mixtures in the range of $0.8<\phi<1.3$, and for the methane-air mixtures in the range of $0.9<\phi<1.2$. Consequently, we may expect a deflagration-to-detonation transition (DDT) to occur in these cases. In fact, a reasonable and conventional parameter to analyze the possibility of DDT and it potential locus is the so-called flame run-up distance - a distance that a flame front propagates from its ignition and until the detonation initiation. In the present formulation, we shall approximate the flame run-up distance as the flame tip position at the instant when its velocity is equal to the local sound speed, $d Z_{t i p} / d t=c_{0}$, although it is recognized that such a definition is not accurate, and the detonation does not occur exactly at the instance when the flame speed in the laboratory flame penetrates the sound barrier. Still these values correlate, and therefore this is a reasonable approximation within the overall frame of this work. Then Eq. (3.25) yields

$$
c_{o}=\left.\frac{d Z_{t i p}}{d t}\right|_{r . u . d}=2(\Theta-1) U_{D L}(t) \frac{R_{f}(t) Z_{r . u . d}}{H^{2}}+\Theta U_{D L}(t),
$$

and the associated flame run-up distance is given by

$$
Z_{\text {r.u.d }}=\frac{\left(c_{o}-\Theta U_{D L}(t)\right) H^{2}}{2(\Theta-1) R_{f}(t) U_{D L}(t)}
$$

In the Eq. (4.2), it should not be confused that run up distance depend on instantaneous time. The time showed here is not instantaneous but it is integrated value which is approximated when the flame tip speed for a given equivalence ratio reaches the associated sound speed for the gas. In this regard, it can be calculated as, first, by approximating Eq. (3.27) equal to associated sound speed for the gas, then after iteration process, integral time value can be found and implemented into Eq. 4.2, in order to calculate run up distance. 
For the equivalence ratio of a methane-air flow exceeding 0.8 , the flame tip velocity in the laboratory reference frame exceeds the sound speed. We can therefore guess that for the equivalence ratios in the range of $0.8<\phi<1.3$ the methane-air flame will trigger a detonation by the time when a flame skirt approaches the sidewall. The result is shown in Fig. 4.6. In fact, this plot identifies the distance the flame propagates before the detonation triggers.

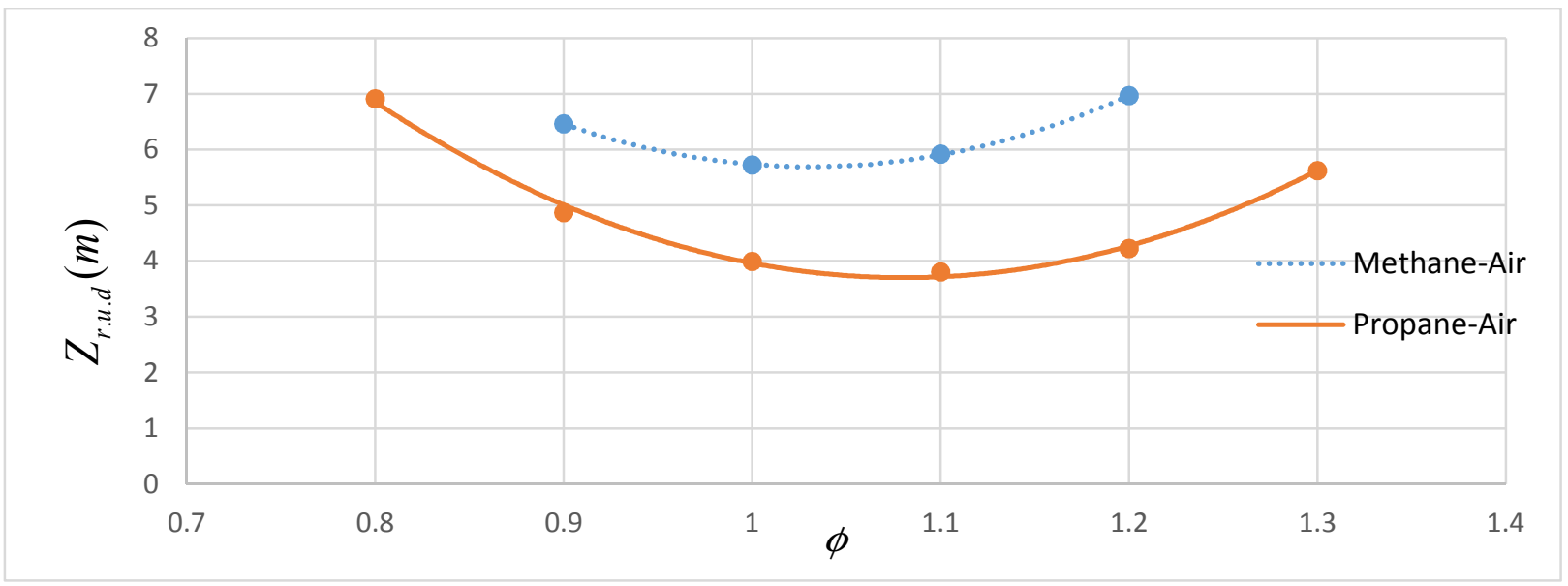

Fig. 4.6: Flame run up distance, $Z_{\text {r.u.d }}$, Eq. (4.2) versus the equivalence ratio $\phi$. The methane-air and propane-air mixtures are shown by the dashed and solid lines, respectively.

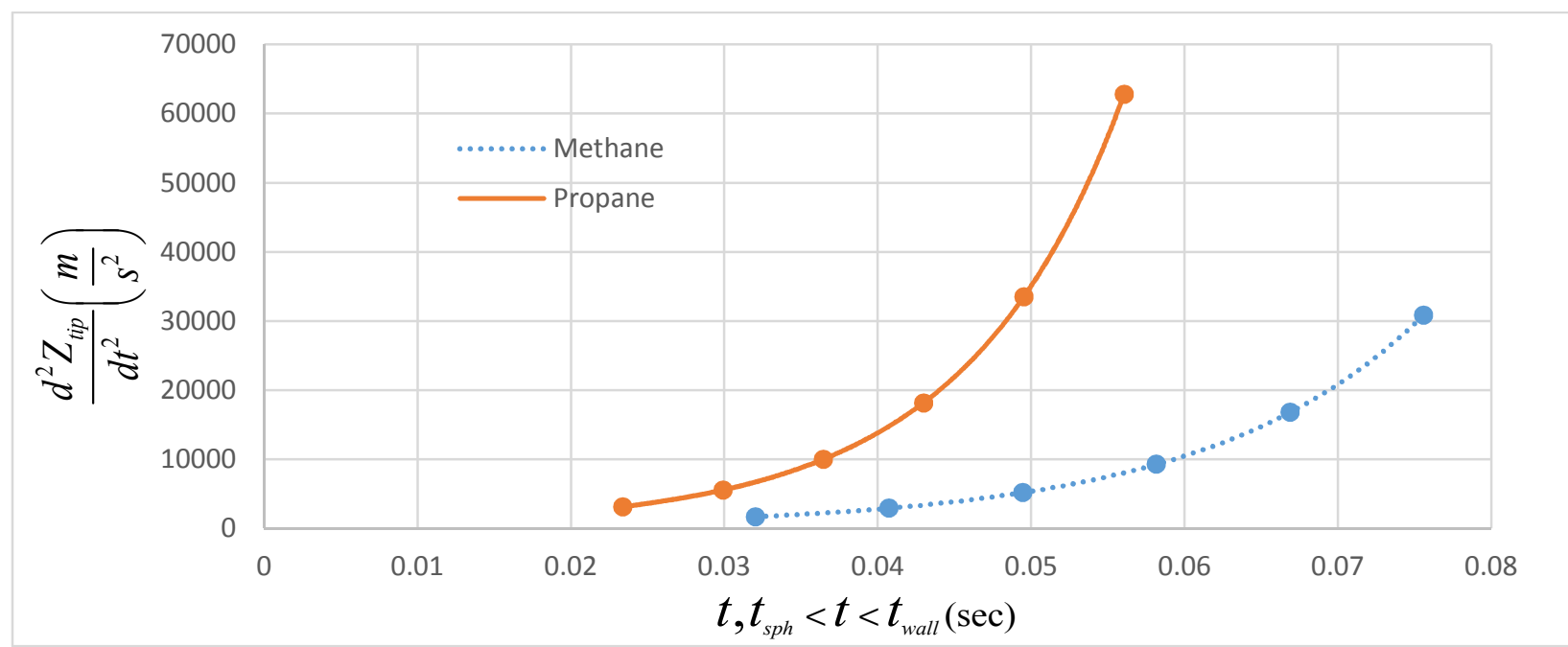

Fig. 4.7: Time Evolution of flame tip acceleration $d^{2} Z_{\text {tip }} / d t^{2}$, Eq. (3.28) within the interval $t_{\text {sph }}<t<t_{\text {wall }}$ between $t_{\text {sph }}$, Eq. (3.23), and $t_{\text {wall }}$, Eq. (3.31). The methane-air and propane-air mixtures are shown by the dashed and solid lines, respectively. 
Figure 4.7 presents the time evolution of the flame tip acceleration, Eq. (3.28), within the time interval $t_{s p h}<t<t_{\text {wall }}$, for the stoichiometric methane-air and propane-air mixtures. It is seen here that by the characteristic time $t_{s p h}$, the flame tip acceleration raises noticeably due to the DarriusLandau instability. Then, by the time $t_{\text {wall }}$, the finger flame scenario amplifies the acceleration by one more order of magnitude. If we compare this acceleration to that due to gravity, $g=9.8 \mathrm{~m} / \mathrm{s}^{2}{ }^{2}$ it will be recognized that the flame-generated acceleration is huge indeed, and it may rise by three orders of magnitude by the time a flame skirt contacts the tunnel wall.

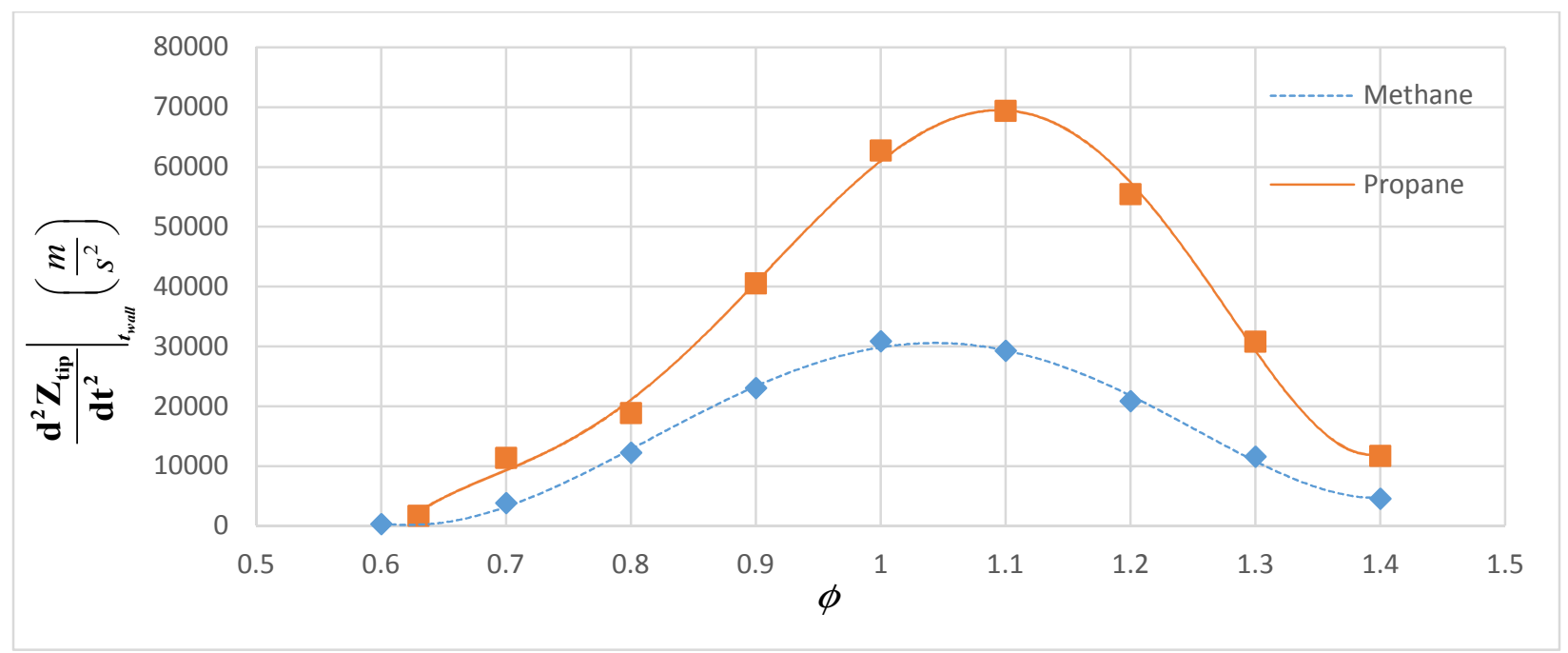

Fig. 4.8: Maximal flame tip acceleration $\left(d^{2} Z_{t i p} / d t^{2}\right)_{\text {wall }}$, Eq. (3.28), taken at the termination time $t_{\text {wall }}$, Eq. (3.29), vs the equivalence ratio $\phi$. The methane-air and propane-air mixtures are shown by the dashed and solid lines, respectively.

Figure 4.8 illustrates the maximal flame tip acceleration achieved for both methane-air and propane-air mixtures at the characteristic time $t_{\text {wall }}$. Equation (3.29) shows that short-in-time flame acceleration before the flame skirt contacted the wall is very strong in either case compared. 


\section{Chapter 5: Effect of Environmental Dust on Flame Propagation in Tunnels}

\subsection{Motivation}

Many substances that are non-flammable in a bulk form become explosive if dispersed as a cloud of fine particles in air. From a practical viewpoint, this can be treated as both a benefit and a hazard, depending on weather we would like to trigger/facilitate combustion for, example in coal fired power plants or suppress/prevent the same specifically in industries that manufacture, transport, process and/or use combustible dusts, accidental dust deflagrations represent a real hazard to both personnel and equipment such as in coal mines. An illustrative example is a recent catastrophic dust explosion in the Upper Branch coal mine (West Virginia, April 5, 2010) that killed 29 miners and is considered as one of the most disastrous dusty mining explosion in the recent US history. Another example is a catastrophe in Soma, Turkey in May 13, 2014 that killed more than 300 miners. Generally, most of coal mine explosions often involve a methane deflagration combined with fugitive coal dust that is collected by the combustion wave as it progresses through the mine. First, the dust deposited on the floor, walls, and ceiling can be lifted up by the pressure blast of the initial methane explosion causing a cloud of dust to be suspended in the air. When the methaneair flame front meets the dust cloud, the coal particles pyrolyze and contribute a volatile vapor to the methane-air mixture. The coal particles can also cause combustion instabilities, which could potentially alter the structure of the premixed flame. However, the interaction between solid combustible dust particles and a gaseous premixed flame have been rarely investigated in combustion literature and are also the focus of current work. 


\subsection{Mathematical Model}

\subsubsection{Effect of equivalence ratio promotion}

Coal dust particles can release volatiles into the gaseous mixture as the result of the elevated particle temperature. It is noted that the volatiles constitutes the addition to the gaseous fuel, which thereby increases the equivalence ratio locally. In order to estimate the fraction of volatiles present in the gaseous mixture, it is necessary to explore the rate of the devolatilization process and quantify such a rate. Overall, there are four possible methods to estimate the devolatilization. 1) The first method is based on the assumption that all volatiles are released when particle reach a given temperature. However, such approach excludes the fact that devolatilization is a transient process. 2) The second method is to treat the particles as liquid droplets where the vaporization can be estimated as discussed by Suard et al. [35]. This method, however, does not capture the non-linear behavior of devolatilization of coal since the droplet evaporation is fairly linear. 3) According to the third method, the devolatilization can be determined by solving a set of Arrhenius-based equations, as described by Solomon and Colket [36]. However this method requires a relatively high computational cost. 4) Finally, within the frame of the fourth method, the devolatilization can be estimated based on a temperature-dependent power-law relation as proposed by Seshadri et al [27]. To be specific, Seshadri et al. [27] suggested the following expression to predict the devolatilization rate of coal particles,

$$
w_{v}^{\prime}=A n_{s} 4 \pi r^{2} T_{s}^{n},
$$

where $w_{v}^{\prime}$ denotes mass of gaseous fuel vaporized per unit volume. The quantities $A$ and $n$ are constants describing the rate of vaporization of particles and the temperature exponent characterizing rate of vaporization of fuel particles, respectively. The particle temperature is denoted as $T_{s}$ and it is approximated as 


$$
T_{s}=\frac{T_{b}+T_{v}}{2},
$$

where $T_{b}$ is the adiabatic flame temperature based on the methane-air equivalence ratio and $T_{v}$ is the temperature at which devolatilization is initiated. For instance, $T_{v} \sim 600 \mathrm{~K}$ for coal particles [37]. The total number of particles per unit volume of mixture is estimated by means of dividing the dust concentration (which is denoted as $c_{s}$ and should not be confused with sound speed defined in Chapter 4 , which is denoted by $c_{o}$ ) by the particle density $\rho_{s}$. The number of particles per unit volume, $n_{s}$, is

$$
n_{s}=\left(c_{s} / \rho_{s}\right) / V_{\mathrm{s}}
$$

The specific heat capacity of the mixture is given by

$$
C=C_{p}+\frac{c_{s} C_{s}}{\rho},
$$

where $C$ is total heat capacity of mixture and should not be confused with the factor $C$ which is given in Chapter $3, C_{p}$ is heat capacity of the gas phase, $C_{s}$ is heat capacity of particles and $\rho$ is the overall density of mixture, which is given by

$$
\rho=\rho_{u}+c_{s},
$$

where $\rho_{u}$ is the density of the gas phase and $c_{s}$ is the concentration of particles. The characteristic time, $t_{r}$, of the particles in preheat vaporization is estimated from Ref. [27] as

$$
t_{r}=\frac{\lambda_{u}}{\rho_{u} S_{L}^{2} C} .
$$

Here $S_{L}$ is the velocity of gaseous flame without particles for given equivalence ratio. The characteristic time is used to estimate the total mass of released volatiles per unit volume

$$
w_{v}=w_{v}^{\prime} t_{r} .
$$


For simplicity, this additional fuel is assumed to be methane $\left(\mathrm{CH}_{4}\right)$, as suggested in Ref. [27] and added to the original gaseous mixture to obtain a new equivalence ratio. The new amount of gaseous fuel in the mixture is denoted as

$$
M_{\text {fuel }}^{m}=M_{C H 4}^{m}+w_{v},
$$

where $M_{C H 4}^{m}$ is the mass of original methane in the mixture, accordingly the new equivalence ratio can be estimated as

$$
\phi_{s}=17.2\left(\frac{M_{\text {fuel }}^{m}}{M_{\text {air }}^{m}}\right),
$$

where the coefficient 17.2 is the mass of methane to mass of air when $\phi=1, M_{\text {air }}^{m}$ is the actual mass of air for given equivalence ratio. With this new equivalence ratio, a new flame temperature $T_{f}^{\prime}$ is estimated by an equilibrium solver GASEQ [38], which allows us predicting the effects of changes in temperature, pressure, and concentration on a system at equilibrium. It states that if a system at equilibrium experiences a change, then the system will shift its equilibrium trying to compensate for the change [38]. The outcome for methane-air premixed flame is shown in Figs. 5.1 and Fig. 5.2, where the new equivalence ratio $\phi$ is presented versus the dust concentration for various equivalence ratios. The associated values of the parameters are presented in the Table 5 for dust particles. Because of the presence of extra fuel in a given locus of the premixture promotes the equivalence ratio. This is justified in Figs. 5.1 and Fig. 5.2, where the modified equivalence ratio is plotted versus the dust particles concentration.

\begin{tabular}{|llll|}
\hline$B(1 / \mathrm{mol})$ & $3.5 \times 10^{6}$ & $\rho_{\mathrm{u}}\left(\mathrm{kg} / \mathrm{m}^{3}\right)$ & 1.135 \\
$K_{u}(\mathrm{~kJ} / \mathrm{ms})$ & $0.52 \times 10^{-4}$ & $\rho_{\mathrm{s}}\left(\mathrm{kg} / \mathrm{m}^{3}\right)$ & 1000 \\
$E(\mathrm{~kJ} / \mathrm{mol})$ & 88.8 & $C_{p}(\mathrm{kj} / \mathrm{kgK})$ & 2.22 \\
$k J(1 / \mathrm{molK})$ & $8.314 \times 10^{-3}$ & $C_{s}(\mathrm{kj} / \mathrm{kgK})$ & 1.26 \\
\hline
\end{tabular}

\section{Table 5: Various parameters of combustible particles [19].}




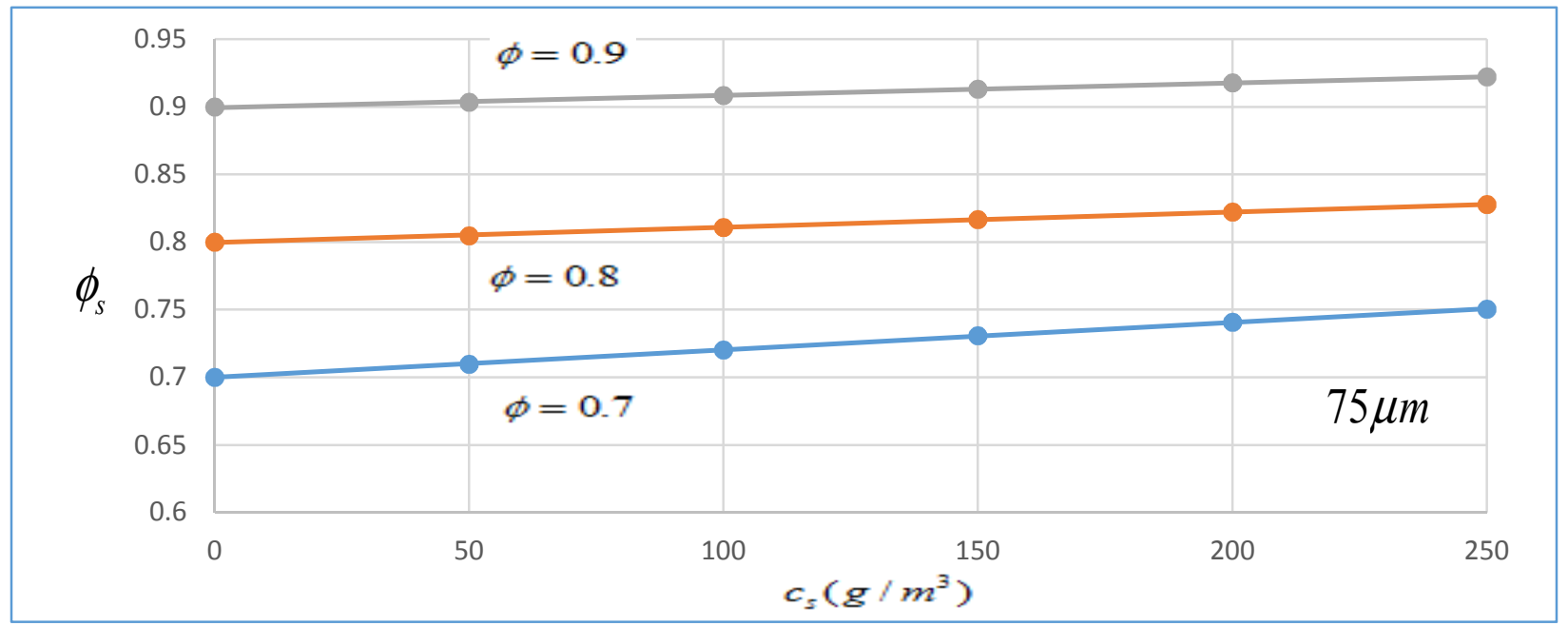

Fig 5.1: The modified equivalence ratio vs the concentration of particles of size 75 microns.

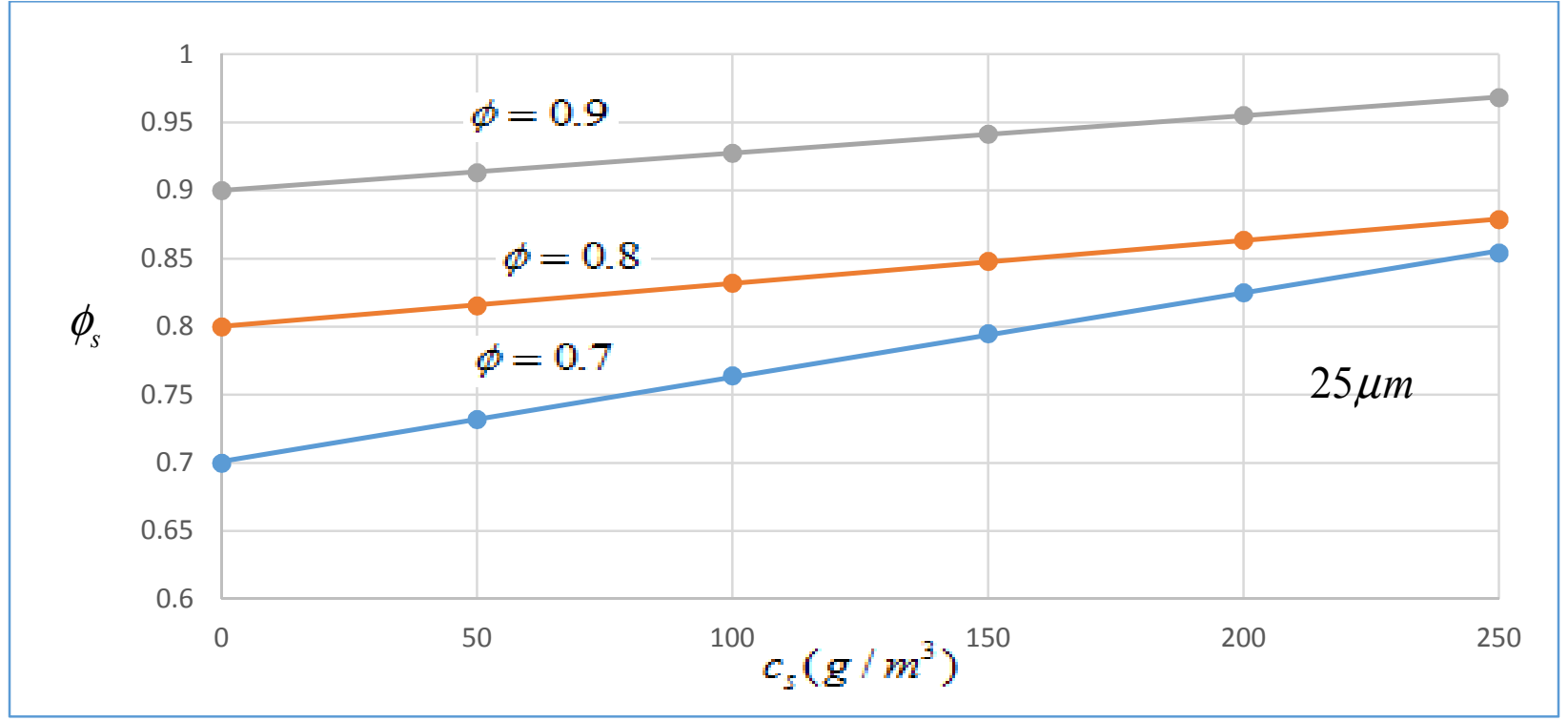

Fig 5.2: The modified equivalence ratio vs the concentration of particles of size 25 microns.

Because of the presence of extra fuel due to the coal particles the equivalence ratio is promoted in the premixture. This is justified in Figs. 5.1 and 5.2 where equivalence ratio plotted versus the dust particles concentration. Consequently the temperature increase in Figs. 5.3 and 5.4 is because of addition of coal particles that devolatilize forming a known gaseous compound, which is assumed 
to be methane. Also, the particles with lesser size promote the temperature increase even better which can be seen in Fig. 5.4. A light increase in the equivalence ratio is seen in Fig. 5.3., which in turn increases the flame temperature calculated using GASEQ equilibrium solver [38] for lean fuel mixtures considered here $(0.6<\phi<1.0)$.

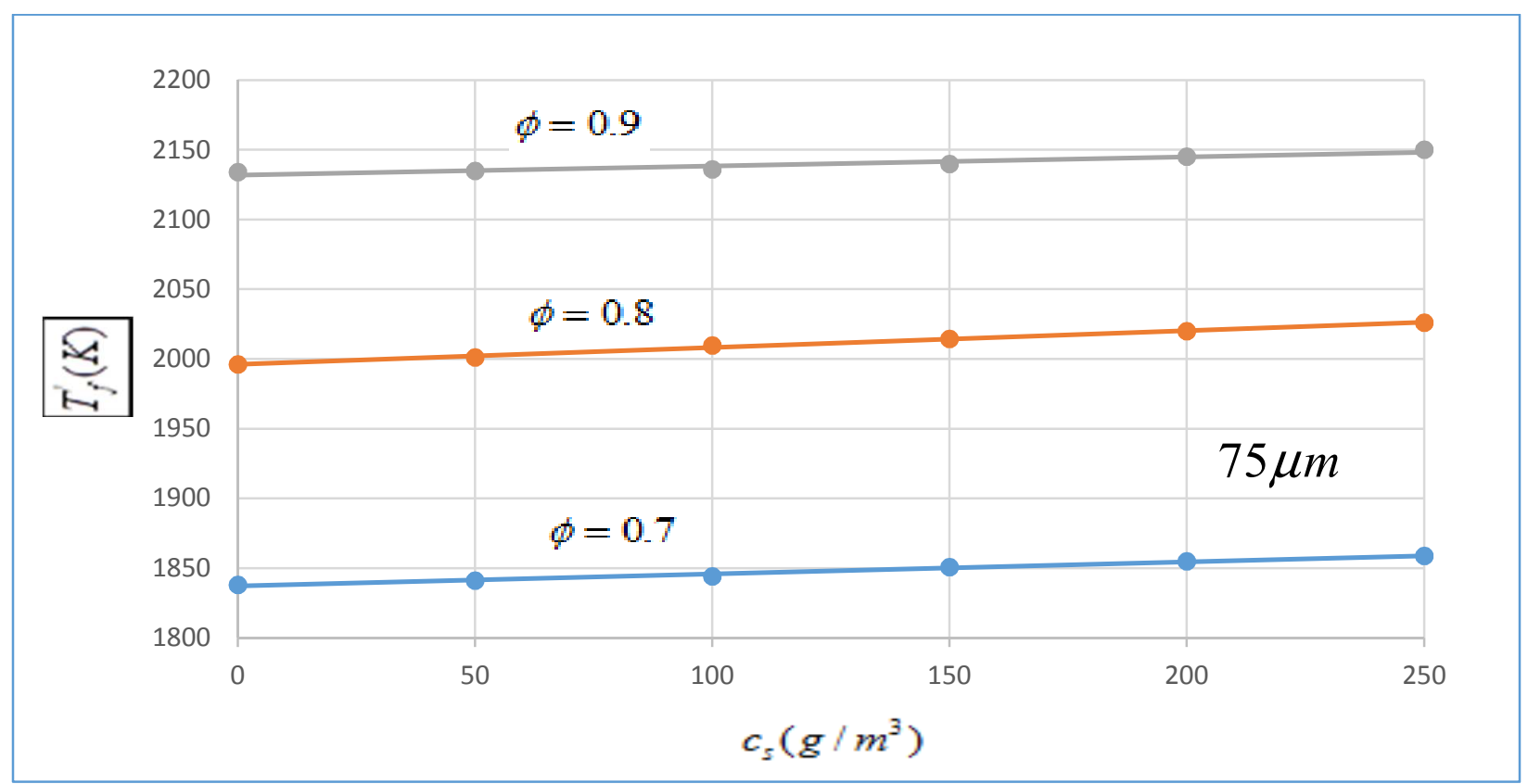

Fig 5.3: The modified flame temperature, Eq. (5.9), versus the dust concentration of particle size 75 microns.

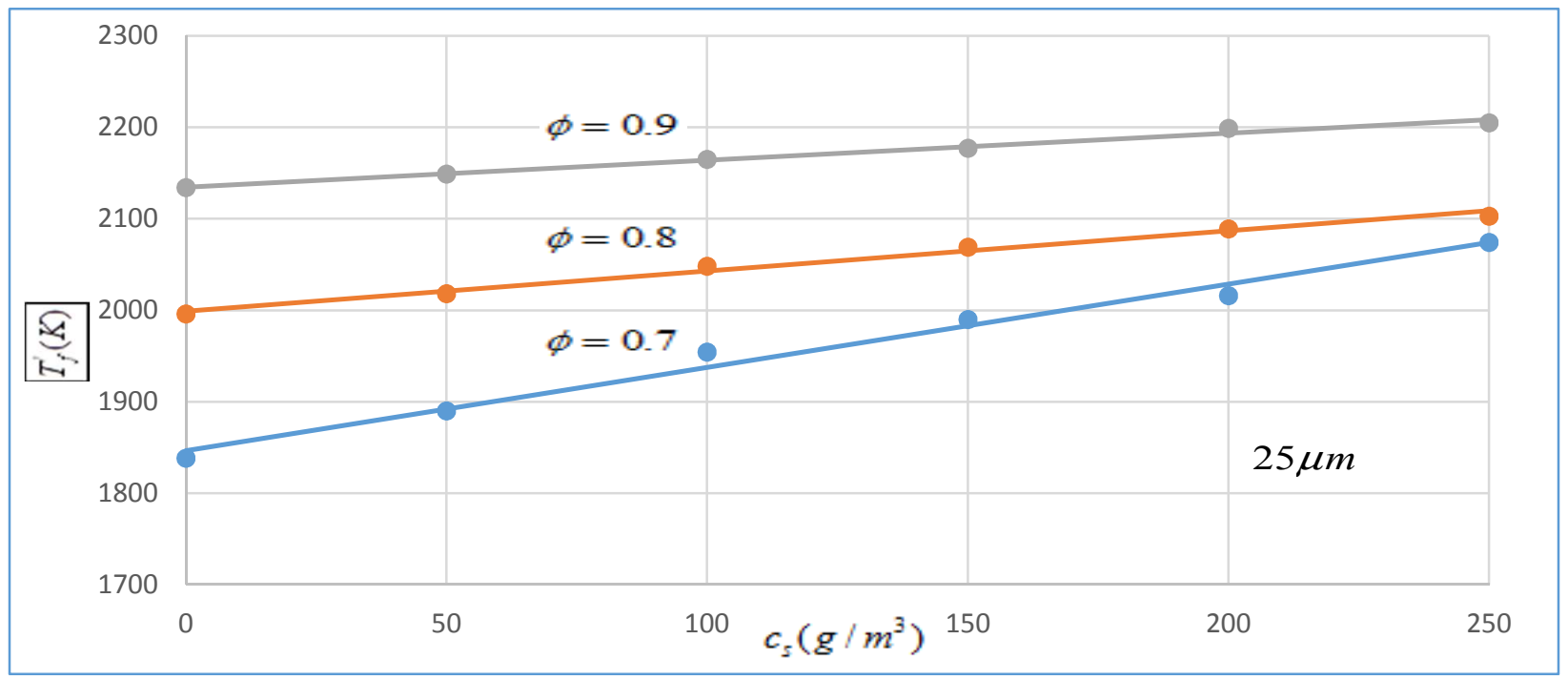

Fig 5.4:The modified flame temperature, Eq. (5.9), versus the dust concentration of particle size 25 microns. 
The corresponding modified flame temperature $T_{f}^{\prime}$ is subsequently used to estimate the "update" in the planar flame speed by means of the Seshadri [27]. Specifically, the dust-induced laminar burning velocity, $S_{L}^{\prime}$, is then calculated as [27]:

$$
S_{L}^{\prime}=\left[\frac{2 B k_{u} \varepsilon^{2}}{\rho_{u} C_{p}} \exp \left(-\frac{E}{R T_{f}^{\prime}}\right)\right]^{1 / 2},
$$

where

$$
\rho_{u}=1.135 \frac{\mathrm{g}}{\mathrm{cm}^{3}}, \quad \varepsilon=\frac{1}{Z_{e}}, \quad Z_{e}=\frac{E\left(T_{f}^{\prime}-T_{u}\right)}{R T_{f}^{\prime 2}} .
$$

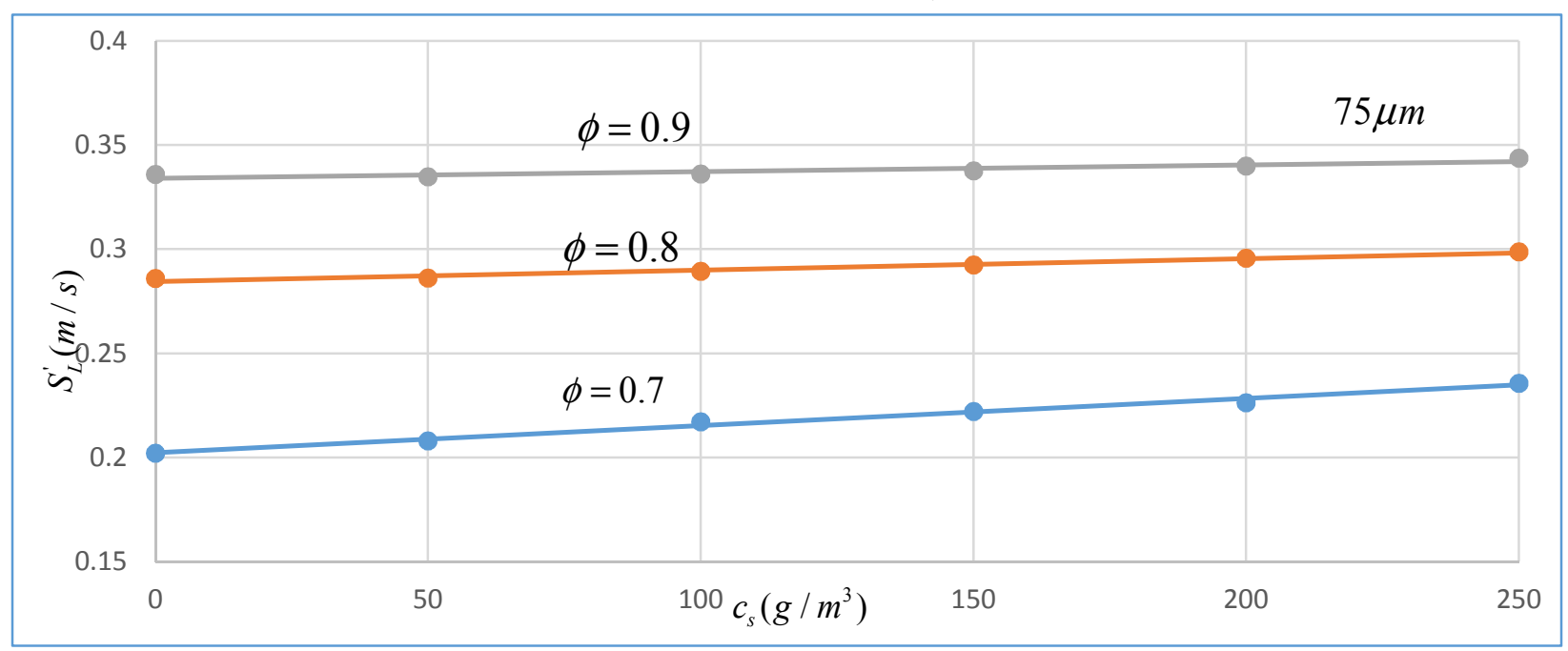

Fig 5.5: The modified burning velocity, Eq. (5.10), vs the dust concentration of particle size 75 microns for different equivalence ratios.

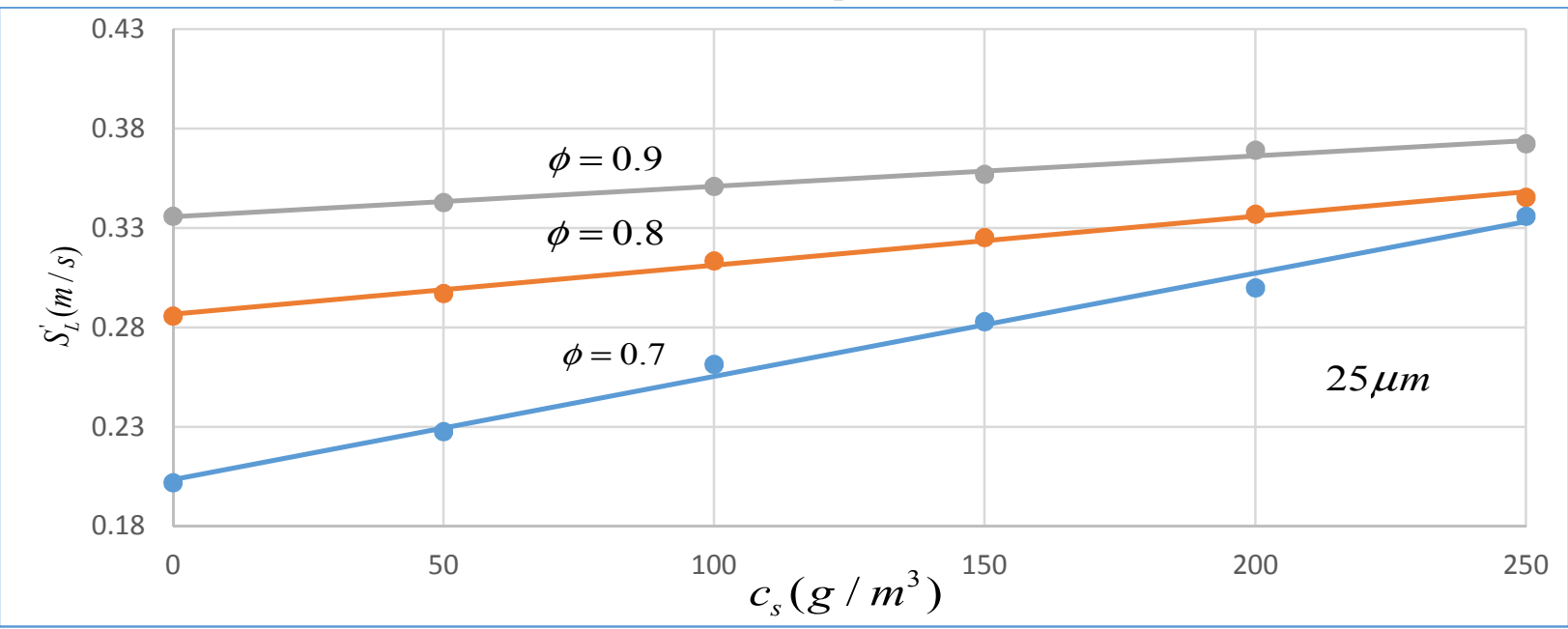

Fig 5.6: The modified flame temperature, Eq. (5.10), vs the dust concentration of particle size 25 microns for different equivalence ratios. 
The calculated laminar burning velocity due to the addition of the coal particles of two different sizes are shown in Figs. 5.5 and 5.6. The promotion in the equivalence ratio due to the temperature rise increases the laminar burning velocity of the flame. The particles of a lesser size promote the burning velocity than those of a bigger size, as shown in Fig. 5.7.

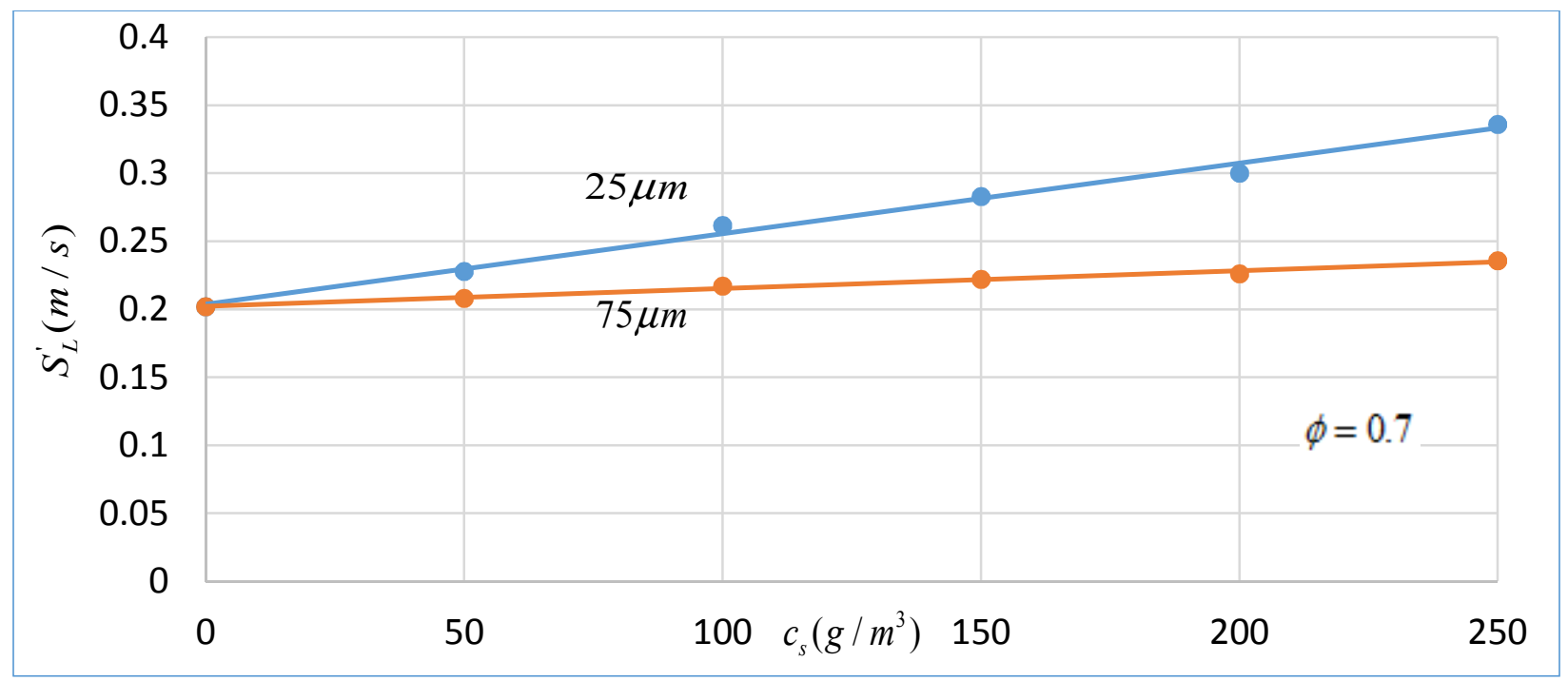

Fig 5.7 Comparison of flame speeds vs the dust concentration of particle size for fixed equivalence ratio.

\subsection{Results and Discussion}

With a modified, dust-induced laminar burning velocity, Eq. (5.10), resulted from the increase in the flame temperature as well as that in the equivalence ratio, we next revisit our formulations and analyses of Chapters 3 and 4. Namely, we employ the modified equivalence ratio and the modified laminar burning velocity to calculate the characteristics of a globally-spherically-expanding and subsequently a finger-like flame front such as the characteristic timing, the flame tip position and velocity, as well as the burning rate. Specifically, the characteristic time instant devoted to a transition from a globally-spherical to a finger-like shape, $t_{s p h}$, can be evaluated as

$$
t \approx\left(\frac{\Theta H}{2 \alpha C}\right)=\frac{n}{\Theta S}\left(\frac{\Theta H}{2 \alpha k}\right),
$$


whereas the instant when the flame skirt contacts the tunnel sidewall, thereby terminating the finger-flame acceleration scenario, in the gaseous-dusty environment reads

$$
t_{\text {wall }}=\left\{\frac{\Theta^{\prime} H}{2 \alpha^{\prime} C^{\prime}} \ln \left(\frac{\Theta^{\prime}+\alpha^{\prime}}{\Theta^{\prime}-\alpha^{\prime}}\right)\right\}^{1 / n}=\frac{n}{\Theta^{\prime} S_{L}^{\prime}}\left\{\frac{\Theta^{\prime} H}{2 \alpha^{\prime} k_{D L}^{n-1}} \ln \left(\frac{\Theta^{\prime}+\alpha^{\prime}}{\Theta^{\prime}-\alpha^{\prime}}\right)\right\}^{1 / n} .
$$

Then the "dusty" counterparts of Eqs. (3.26) - (3.28) acquire the form

$$
\begin{aligned}
& Z_{t i p}=\frac{\Theta^{\prime} H}{2 \alpha^{\prime}} \sinh \left(2 \frac{\alpha^{\prime}}{\Theta^{\prime}} \frac{C^{\prime} t^{n}}{H}\right)=\frac{H}{2} \sqrt{\frac{\Theta^{\prime}}{\Theta^{\prime}-1}} \sinh \left(2 \sqrt{\frac{\Theta^{\prime}-1}{\Theta^{\prime}}} \frac{k_{D L}^{n-1}}{H}\left(\frac{\Theta^{\prime} S_{L}^{\prime}}{n}\right)^{n} t^{n}\right), \\
& \frac{d Z_{t i p}}{d t}=n C^{\prime} t^{n-1} \cosh \left(2 \frac{\alpha^{\prime} C^{\prime} t^{n}}{\Theta^{\prime} H}\right), \\
& \frac{d^{2} Z_{t i p}}{d t^{2}}=n C^{\prime} t^{n-2}\left(2 \frac{\alpha^{\prime} C^{\prime}}{\Theta^{\prime} H} n t^{n} \sinh \left(2 \frac{\alpha^{\prime} C^{\prime} t^{n}}{\Theta^{\prime} H}\right)+(n-1) \cosh \left(2 \frac{\alpha^{\prime} C^{\prime} t^{n}}{\Theta^{\prime} H}\right)\right) .
\end{aligned}
$$

The modified laminar burning velocity is implemented into the formulation of spherical and finger flame scenarios to investigate effect of the equivalence ratio promotion at different dust concentration values. So, the formulation of Chapter 3 is extended to the new parameters obtained after the fuel addition, which changes the equivalence ratio, gas expansion rate and the flame speed because of the increase in flame temperature.

In Fig. 5.8, the evolution of flame tip position, Eq. (5.15), at the time $t_{\text {wall }}$, Eq. (5.13), is plotted. We can see the shift in the flame tip position when the finger flame scenario stops. This shift in position is observed because of the addition of particles up to $250\left(\mathrm{~g} / \mathrm{m}^{3}\right)$ with size of 75 microns and 25 microns. In Fig. 5.9, the characteristic time $t_{s p h}$, Eq. (5.12), for flames with combustible particles is less than that without particles for a given equivalence ratio. So, methane-air flames with particles exhibits globally-spherical (cellular) shape of an expanding flame even earlier as 
compared to methane-air flames without particles. The characteristic time $t_{\text {wall }}$, Eq. (5.13), will also get promoted to occur before for methane-air flames with particles as compared to those without particles. Also, the difference in size promotes the characteristic time to occur even earlier for the smaller size of particles.

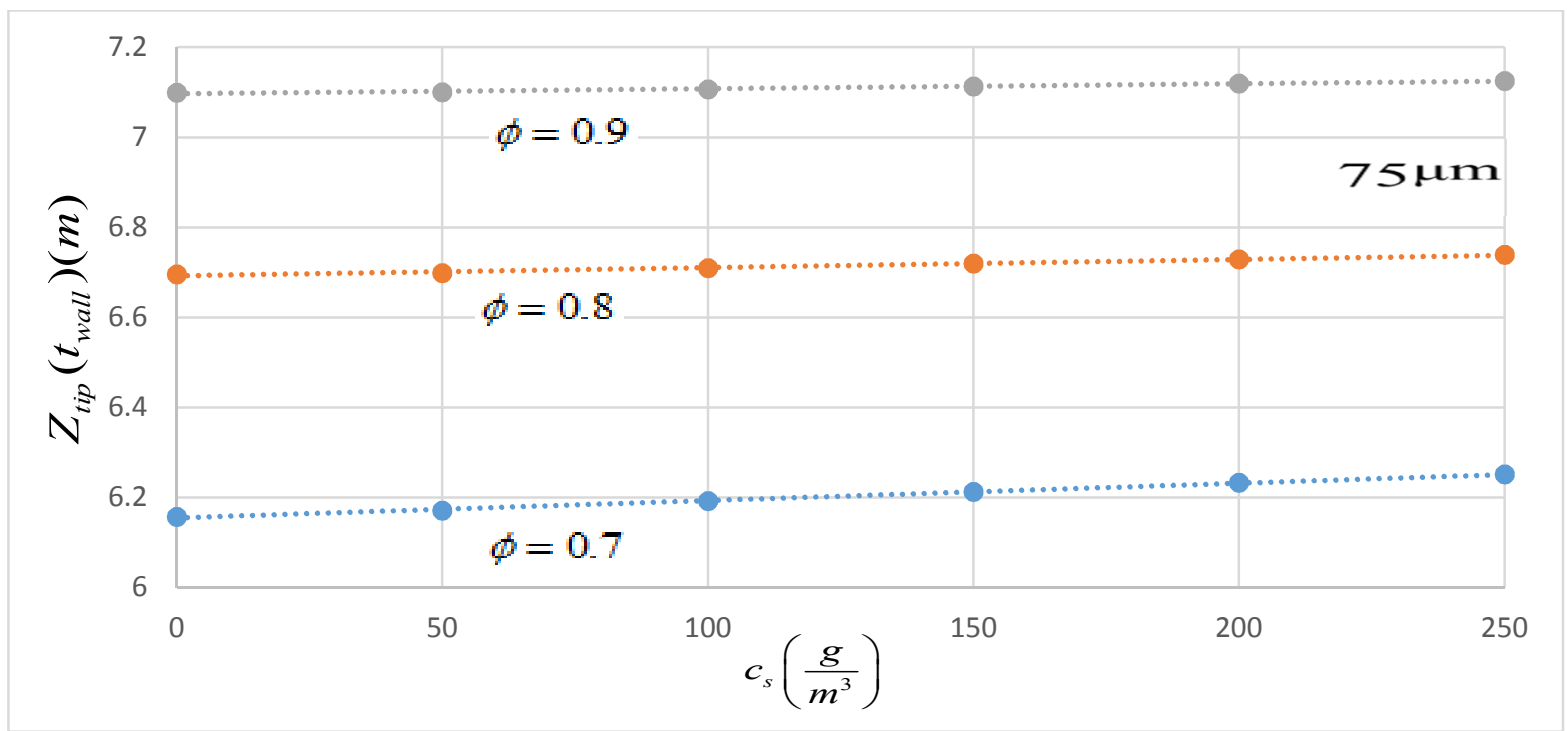

Fig 5.8: Flame tip position, Eq. (5.15), at the time $t_{\text {wall }}$ given by Eq. (5.13), vs the concentration of coal particles for a set of fixed equivalence ratios.

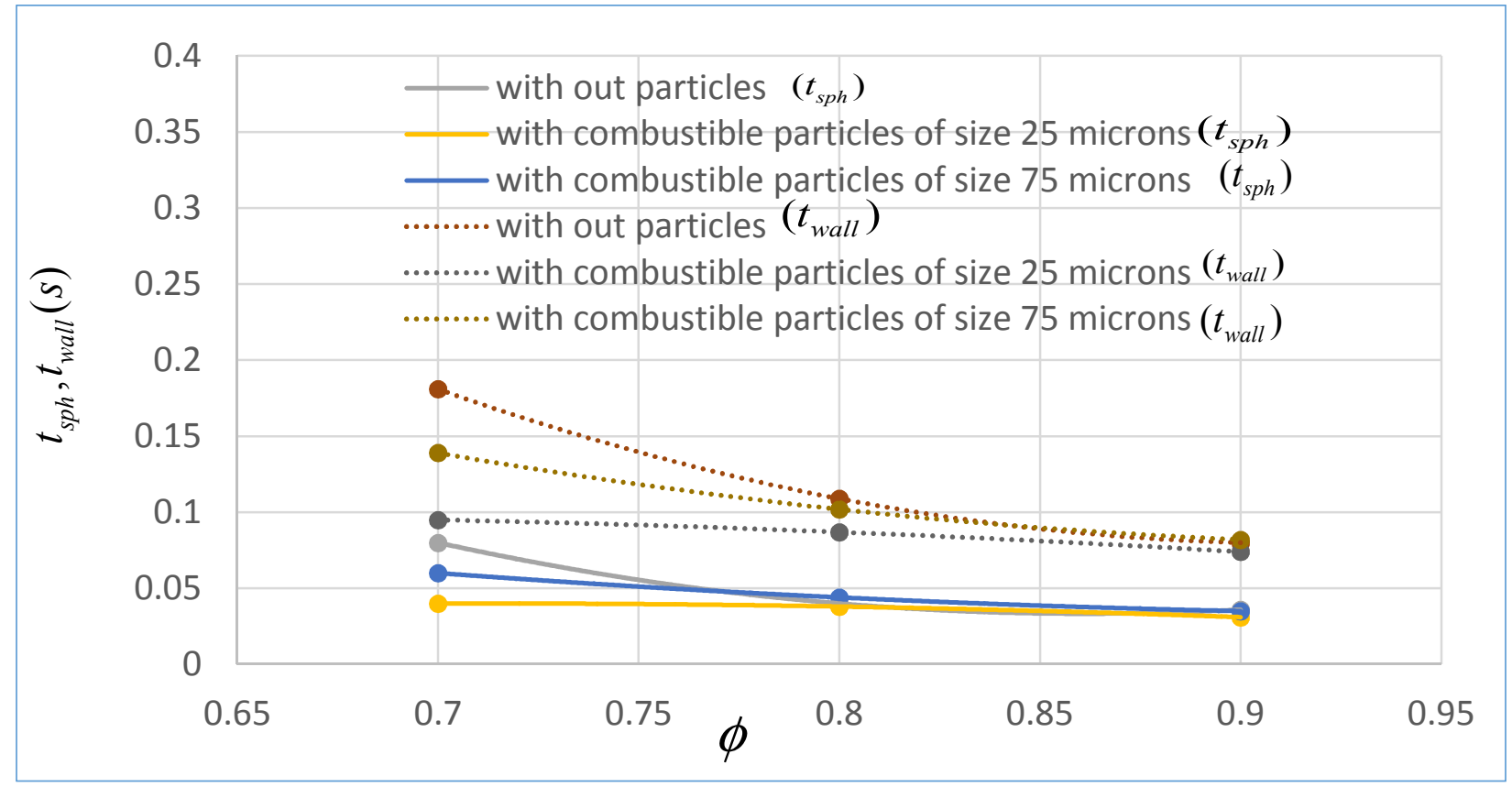

Fig. 5.9: The time limitations of the finger flame acceleration, $t_{s p h}$ of Eq. (5.12) and $t_{\text {wall }}$ of Eq. (5.13), versus the equivalence ratio $\phi$ for methane-air mixture with and without dust particles. 


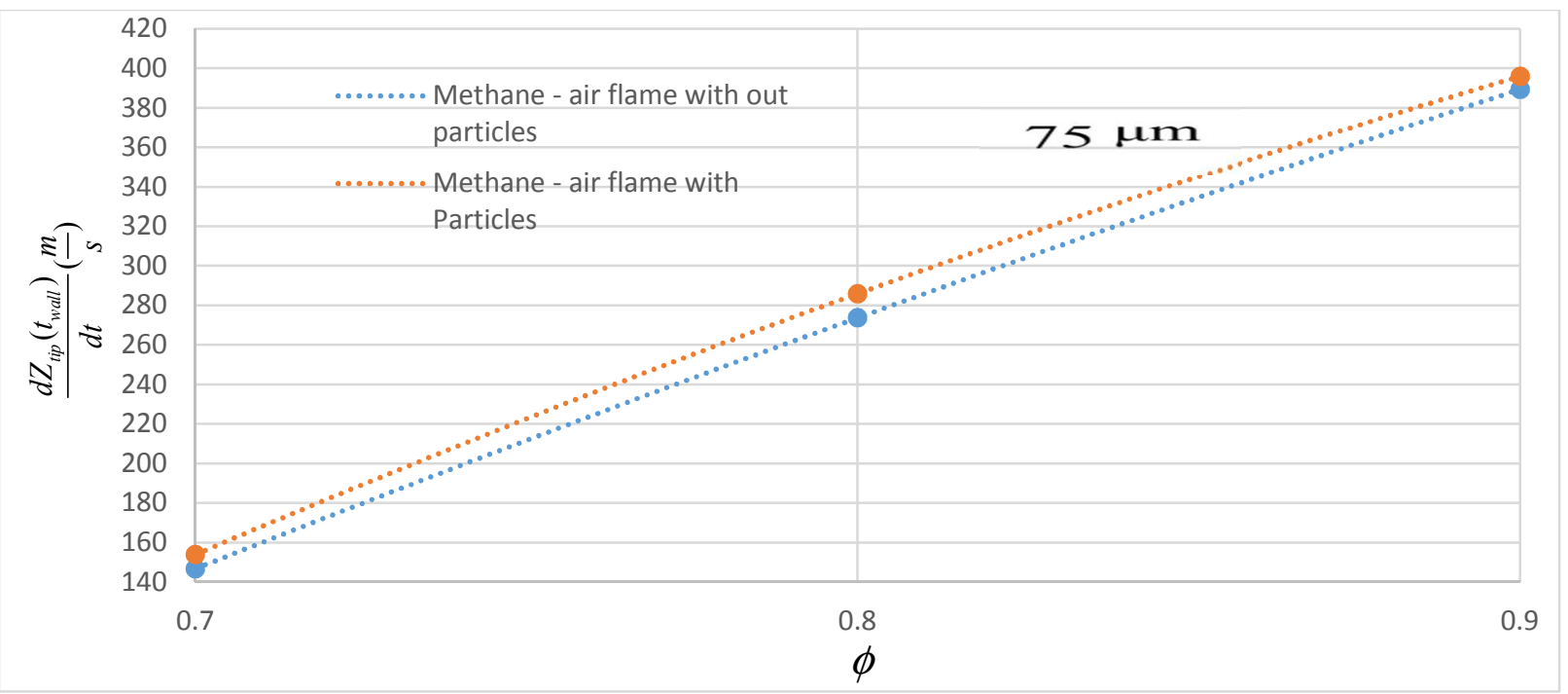

Fig. 5.10: The maximal flame tip velocity $\left(d Z_{\text {tip }} / d t\right)_{\text {wall }}$, Eq. (5.16), at $t_{\text {wall }}$, Eq. (5.14), vs $\phi$ for the methane-air mixture without particles and that with particles of size 75 microns.

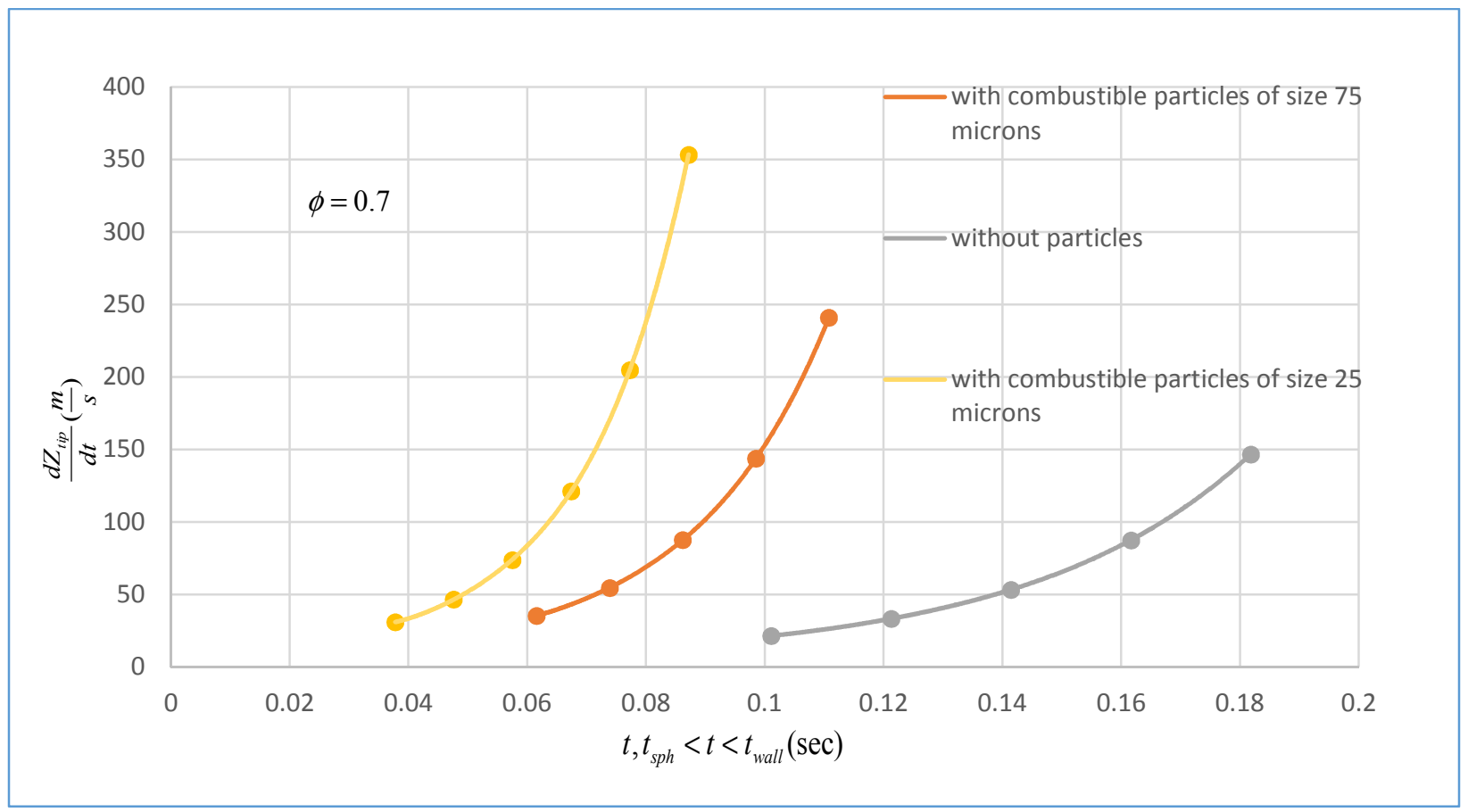

Fig. 5.11: Evolution of flame tip velocity $d Z_{t i p} / d t$, Eq. (5.16), vs the time interval between $t_{s p h}$, Eq. (5.13), and $t_{\text {wall }}$, Eq. (5.14), $t_{s p h}<t<t_{\text {wall }}$. The methane-air with particles and methane-air without particles mixtures are shown for the lean fuel ratio $\phi=0.7$. 
Similar to Fig. 4.5, the maximal flame tip velocity decreases for $0.6<\phi<1.4$, but here, in Fig. 5.10, the maximal flame tip velocity $\left(d Z_{\text {tip }} / d t\right)_{\text {wall }}$, Eq. (5.16), is shown for the lean methaneair mixture $(0.7<\phi<0.9)$ with and without particles. Eventually, Fig. 5.11 presents the time evolution of the flame tip velocity, Eq. (5.16), for the lean methane-air fuel, $\phi=0.7$, with and without combustible particles. It is seen here that the addition of combustible particles to the fuel promotes the flame speed and therefore the termination time $t_{\text {wall }}$ reduces. Anyway, similar to Chapter 3, the flame velocity is increased by two orders of magnitude. Indeed the flame velocity is also increasing with addition of fuel from the coal particles thereby making the fuel rich. 


\section{Chapter 6: Effect of Inert Particles}

\subsection{Mathematical model}

In contrast to combustible (say, coal) particles, inert (say, sand) particles will act as a heat sink as they absorb the heat from the flame and thereby decrease the flame temperature. This effect will be considered in the following section to develop an expression for such a reduced flame temperature. In order to estimate this effect, first, the heat released for flame without dust has to be calculated. For a lean or stoichiometric methane-air mixture, the chemical reaction for combustion with equivalence ratio $\phi$ is given by

$$
\frac{\phi}{2} \mathrm{CH}_{4}+\left(\mathrm{O}_{2}+3.76 \mathrm{~N}_{2}\right) \Rightarrow \frac{\phi}{2} \mathrm{CO}_{2}+\phi \mathrm{H}_{2} \mathrm{O}+3.76 \mathrm{~N}_{2}+2(1-\phi) \mathrm{O}_{2} .
$$

The heat consumed to raise the temperature for $\phi / 2$ moles of methane and 4.76 moles of air, is given by [27]

$$
\left[\left(T_{b}-T_{u}\right) \sum C_{p} n_{\text {product }}\right] .
$$

With the assumption that the entire heat release is used to raise the temperature of the mixture, the heat release rate of the methane-air premixed flame for a given flow of air and a given equivalence ratio is calculated as

$$
Q=\left[\left(T_{b}-T_{u}\right) \sum C_{p} n_{\text {product }}\right] \frac{n_{\text {air }}}{4.76},
$$

where $n_{\text {product }}$ is the number of moles of the products that depends on the equivalence ratio $\phi$. Assuming that the flame with particles releases the same amount of heat while it is also influenced by the temperature rise of particles, a modified flame temperature can be estimated using the energy conservation law

$$
Q=\left[\left(T_{b}-T_{u}\right) \sum C_{p} n_{\text {product }}\right] \frac{n_{\text {air }}}{4.76}+n_{s}^{\prime} C_{s}\left(T_{f}^{\prime \prime}-T_{u}\right) .
$$


Rearranging the terms of Eq. (6.4), we express the new flame temperature, $T_{f}^{\prime \prime}$, in the form

$$
T_{f}^{\prime \prime}=\frac{Q}{\frac{n_{\text {air }}}{4.76} \sum C_{p} n_{\text {product }}+n_{s}^{\prime} C_{s}}+T_{u},
$$

where $n_{s}^{\prime}$ is the number of moles of particle per unit volume passing through the flame, and it is calculated as

$$
n_{s}^{\prime}=\frac{c_{s}}{M_{c}} .
$$

The new flame temperature, accounting for the heat sink effect, is estimated using the equilibrium solver GASEQ [38], and it indicates a continous decrease in the flame temperature with the addition of sand. The corresponding flame temperature is then used to estimate the new laminar burning velocity, $S_{L}^{\prime \prime}$, using the model developed by Seshadri et al. [27]:

$$
S_{L}^{\prime \prime}=\left[\frac{2 B k_{u} \varepsilon^{2}}{\rho_{u} C_{p}} \exp \left(-\frac{E}{R T_{f}^{\prime \prime}}\right)\right]^{1 / 2},
$$

where

$$
\rho_{u}=1.135 \mathrm{~g} / \mathrm{cm}^{3}, \quad \varepsilon=1 / Z e, \quad Z e=\frac{E\left(T_{f}^{\prime \prime}-T_{u}\right)}{R T^{\prime 2}} .
$$

The associated modifications are presented in Figs. 6.1 - 6.4. Specifically, Figs. 6.1 and 6.2 illustrates the decreasing trend in the flame temperature for a methane-air flame with addition of inert particles of a concentration ranging from 0 till $250\left(\mathrm{~g} / \mathrm{m}^{3}\right)$ with the size of particles being 75 microns and 120 microns. As stated above, inert particles decrease the flame temperature which is resulted increase in the flame speed. Such a reduction in the flame burning velocity is also seen in Figs. 6.3 and 6.4, where the modified planar flame speed, induced by the inert dust, is plotted versus the particle concentration. To be specific, the decrease in the flame temperature and propagation velocity is shown for the lean mixture-air in the range of $\phi=0.7-0.9$. 


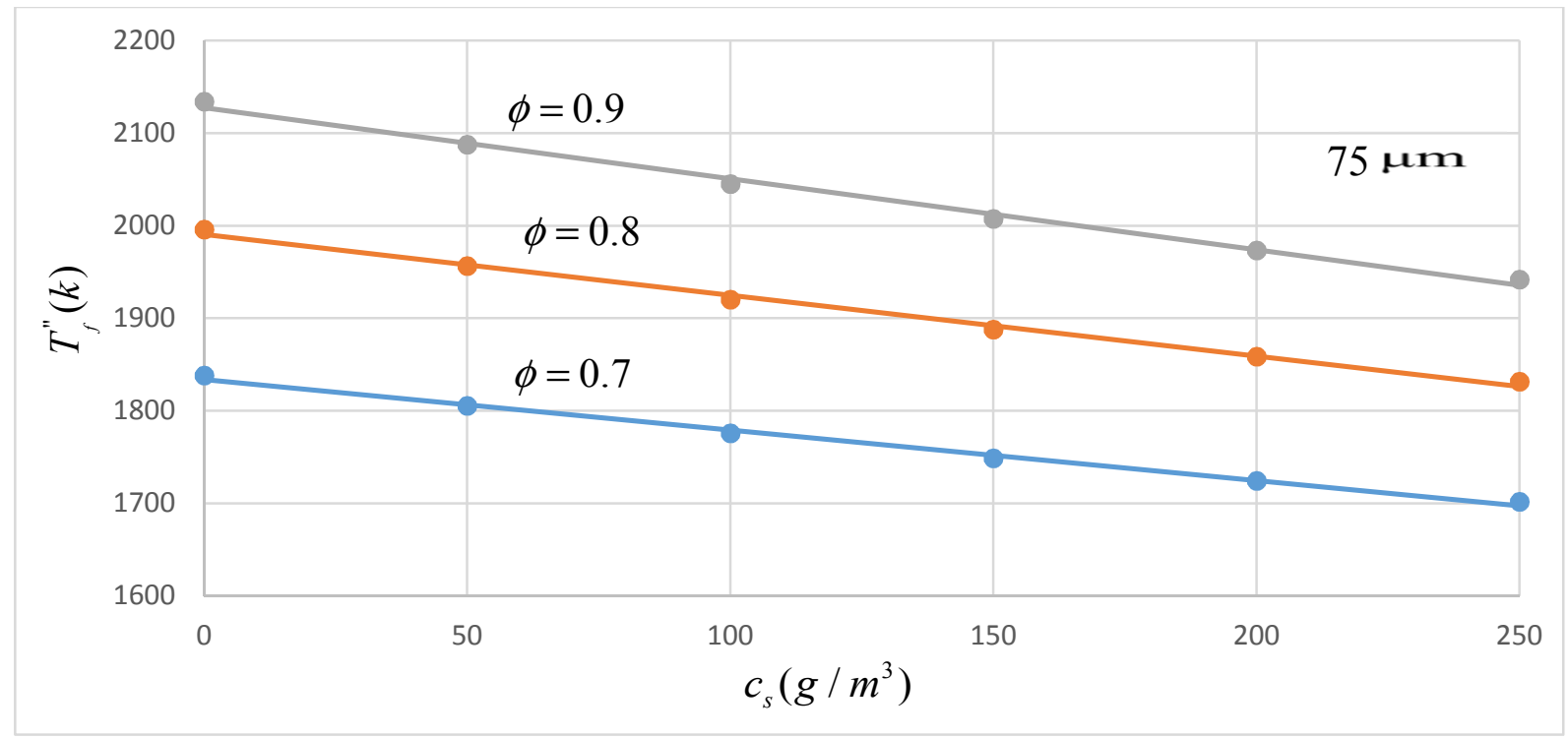

Fig 6.1: Decrease in flame temperature $T_{f}^{\prime \prime}$, Eq. (6.5), versus concentration of particles $c_{s}$ with size of 75 microns.

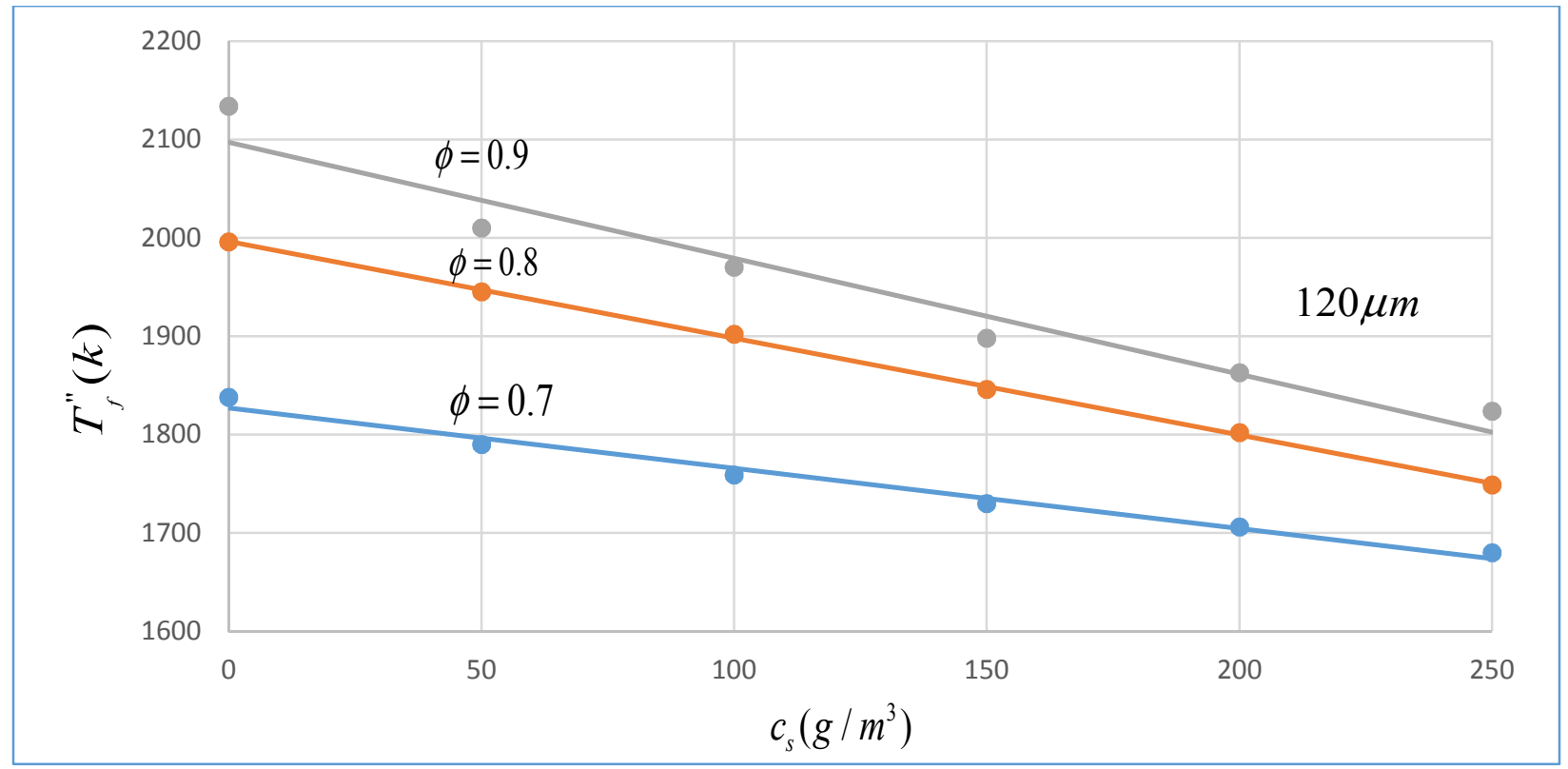

Fig 6.2: The decrease in the flame temperature $T_{f}^{\prime \prime}$, Eq. (6.5), vs the concentration of particles $C_{S}$ for the particles of size 120 microns. 


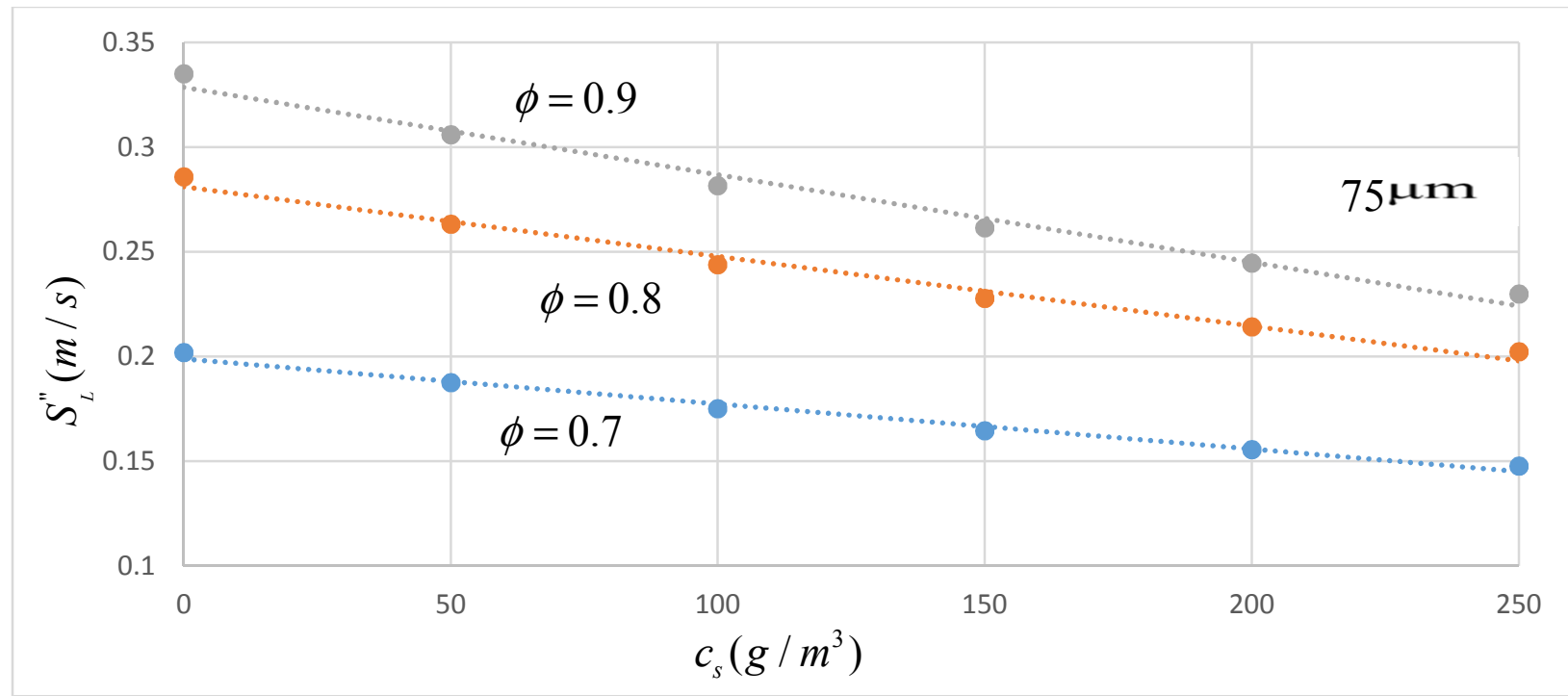

Fig 6.3: Decrease in the burning velocity $S_{L}^{\prime \prime}$, Eq. (6.7), versus concentration of particles $c_{S}$ with size of 75 microns.

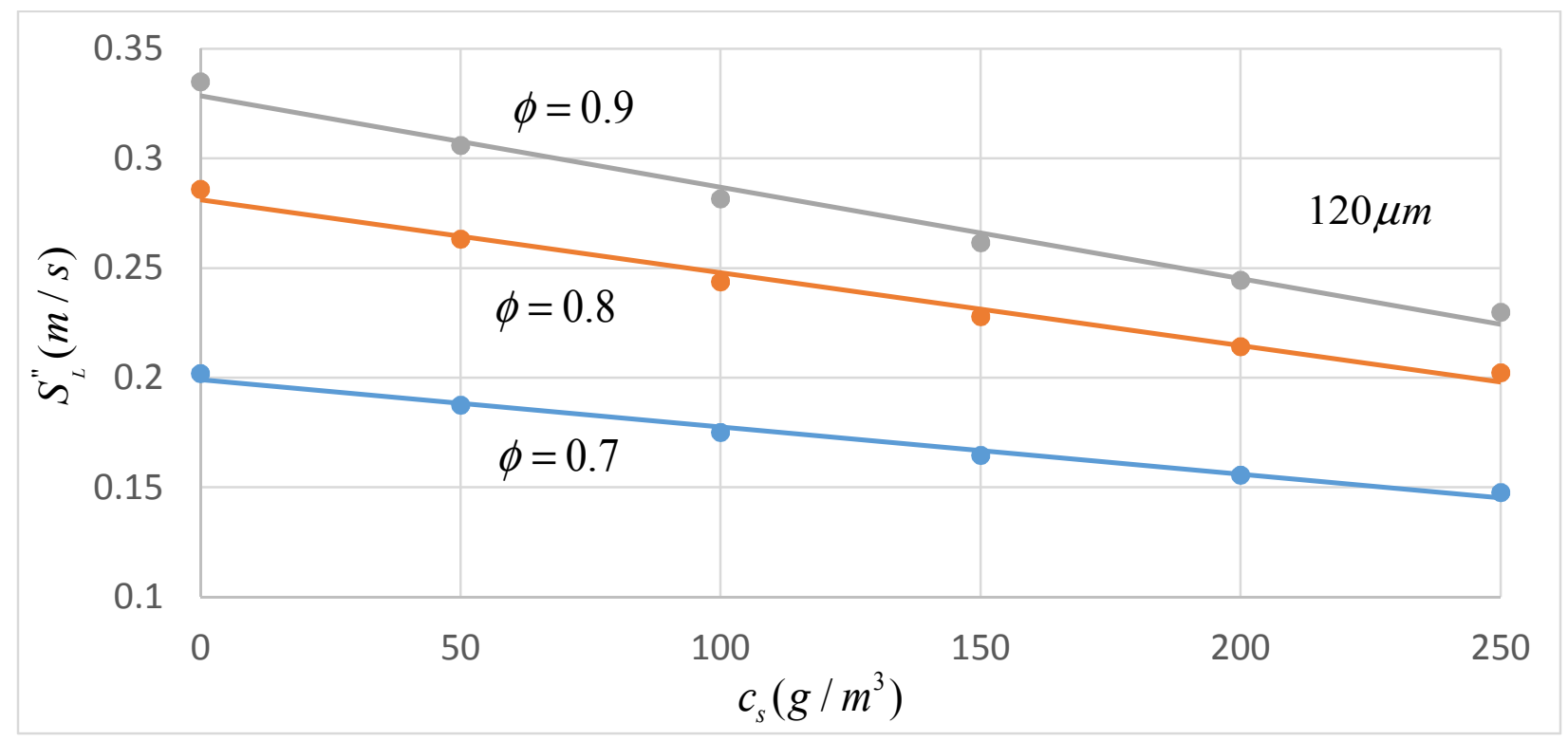

Fig 6.4: Decrease in the burning velocity $S_{L}^{\prime \prime}$, Eq. (6.7), vs concentration of particles $c_{s}$ with size of 120 microns.

The calculated laminar burning velocity due to the addition of inert particles of two different sizes is shown in Figs. 6.3 and 6.4. The temperature decreases due to the heat sink effect of particles, which in turn, reduces the laminar burning velocity. The particles of larger size diminish the laminar burning velocity as compared to the particles of smaller size, see Figs. 6.5 and 6.6, ultimately leading to the mitigation of even termination of a fire; 


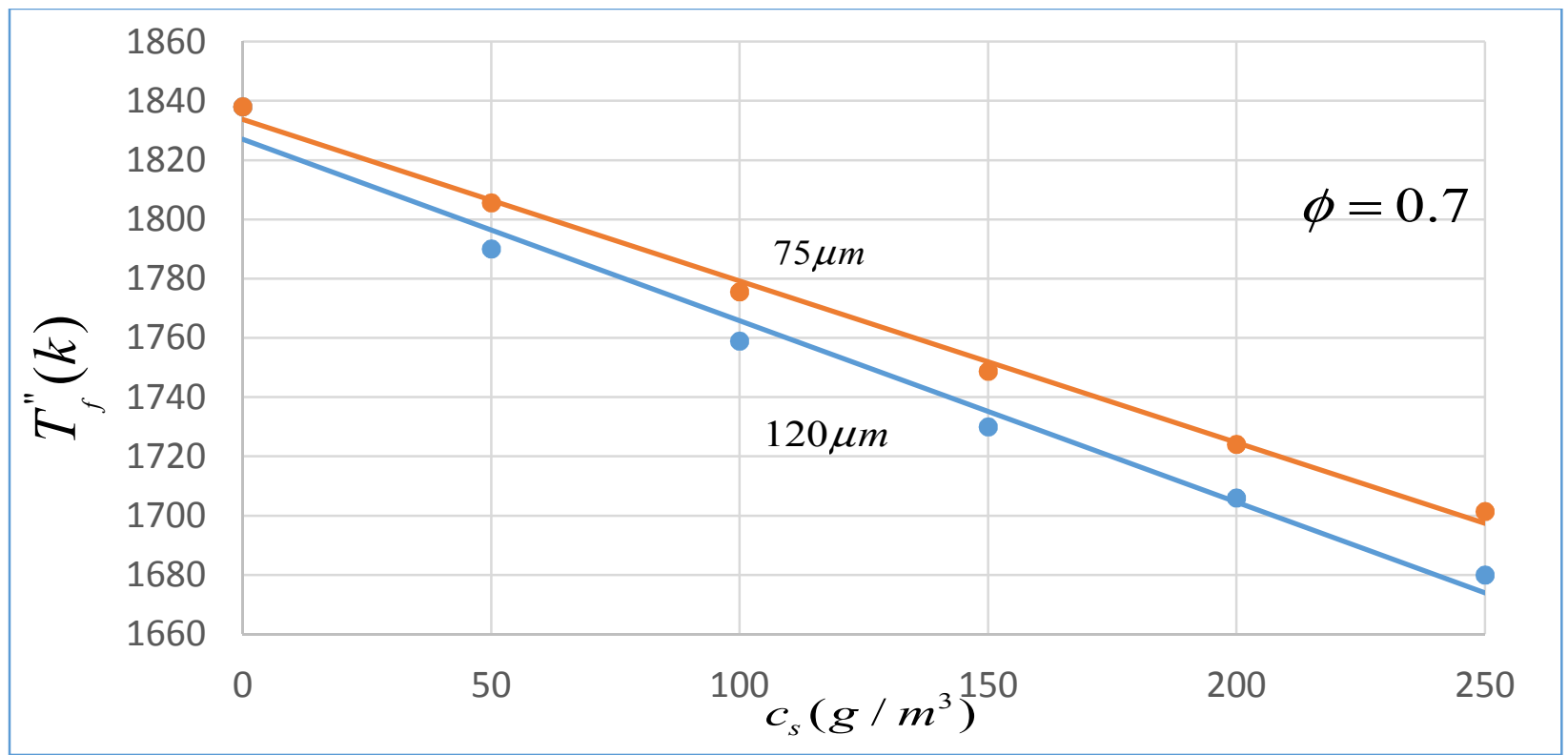

Fig. 6.5: The flame temperature $T_{f}^{\prime \prime}$ with the dust concentration for the particles of size 75 and 120 microns and fixed equivalence ratio $\phi=0.7$.

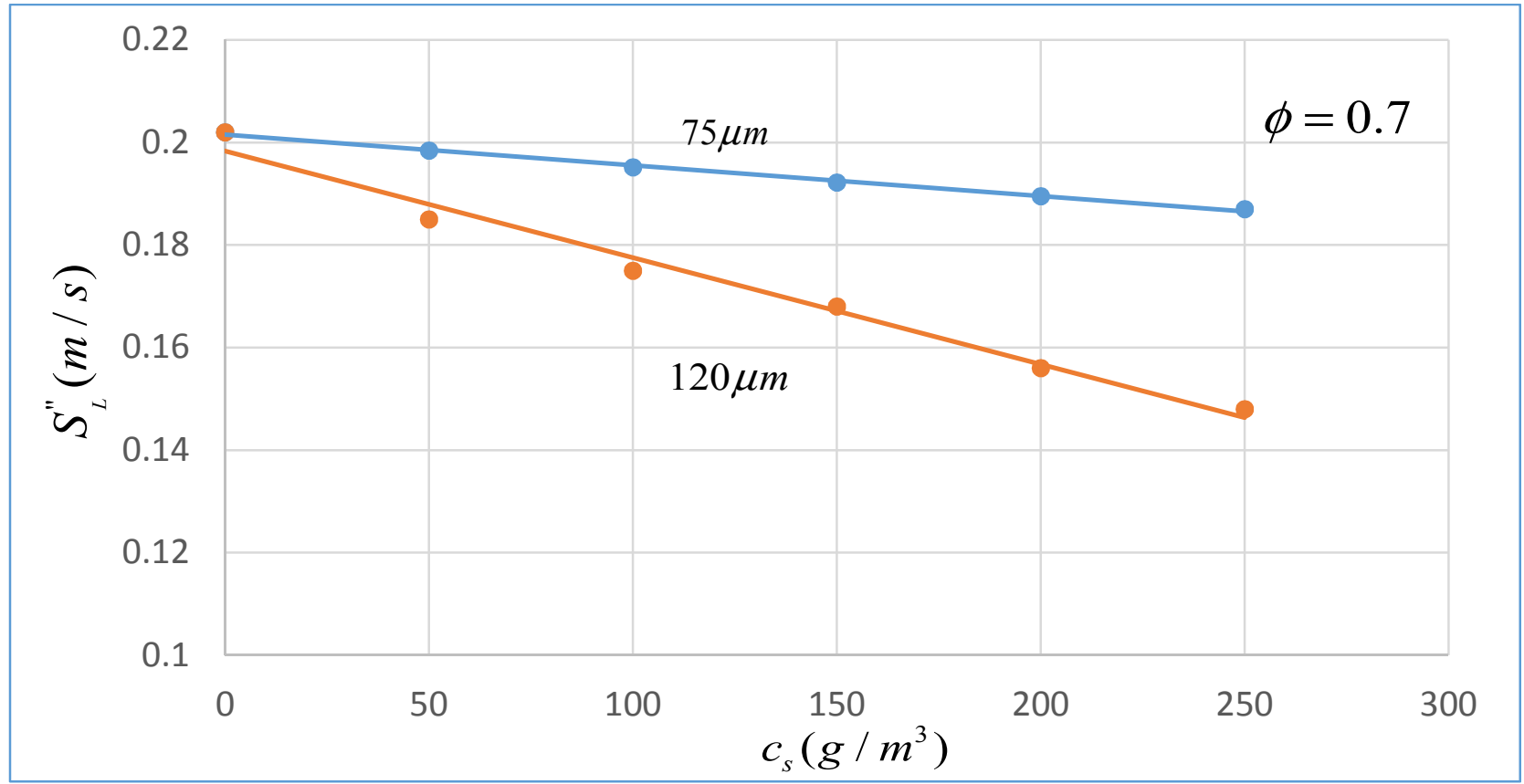

Fig. 6.6: The laminar flame speed $S_{L}^{\prime \prime}$ with the dust concentration for the particles of size 75 and 120 microns and fixed equivalence ratio $\phi=0.7$. 


\subsection{Results and Discussion}

New laminar burning velocity obtained due to the decrease in the flame temperature and the equivalence ratio changes the spherically-expanding and the finger flame scenarios. As we employ the new equivalence ratio and new laminar burning velocity to the formulations obtained in Chapters 3-5, all the parameters, which are functions of the equivalence ratio and the laminar burning velocity will change, and there will be a new estimations for the key stages such as characteristic timing, the flame tip position and velocity, and the burning rate. The "inert" counterparts of Eqs. (3.26) - (3.28) acquire the form

$$
\begin{aligned}
& Z_{t i p}=\frac{\Theta H}{2 \alpha} \sinh \left(2 \frac{\alpha}{\Theta} \frac{C^{\prime \prime} t^{n}}{H}\right)=\frac{H}{2} \sqrt{\frac{\Theta}{\Theta-1}} \sinh \left(2 \sqrt{\frac{\Theta-1}{\Theta}} \frac{k_{D L}^{n-1}}{H}\left(\frac{\Theta S_{L}{ }^{\prime \prime}}{n}\right)^{n} t^{n}\right), \\
& \frac{d Z_{t i p}}{d t}=n C^{\prime \prime} t^{n-1} \cosh \left(2 \frac{\alpha C^{\prime \prime} t^{n}}{\Theta H}\right), \\
& \frac{d^{2} Z_{t i p}}{d t^{2}}=n C^{\prime \prime} t^{n-2}\left(2 \frac{\alpha C^{\prime \prime}}{\Theta H} n t^{n} \sinh \left(2 \frac{\alpha C^{\prime \prime} t^{n}}{\Theta H}\right)+(n-1) \cosh \left(2 \frac{\alpha C^{\prime \prime} t^{n}}{\Theta H}\right)\right),
\end{aligned}
$$

with the characteristic timings

$$
\begin{aligned}
& t_{\text {sph }} \approx\left(\frac{\Theta H}{2 \alpha C^{\prime \prime}}\right)^{1 / n}=\frac{n}{\Theta S_{L}^{\prime \prime}}\left(\frac{\Theta H}{2 \alpha k_{D L}^{n-1}}\right)^{1 / n}, \\
& t_{\text {wall }}=\left\{\frac{\Theta H}{2 \alpha C^{\prime \prime}} \ln \left(\frac{\Theta+\alpha}{\Theta-\alpha}\right)\right\}^{1 / n}=\frac{n}{\Theta S_{L}^{\prime \prime}}\left\{\frac{\Theta H}{2 \alpha k_{D L}^{n-1}} \ln \left(\frac{\Theta+\alpha}{\Theta-\alpha}\right)\right\}^{1 / n} .
\end{aligned}
$$

In Fig. 6.7, the time limitations of the finger flame acceleration for a methane-air flame with inert particles at equivalence ratio $\phi=0.7-0.9$ are showed. The characteristic times, $t_{\text {wall }}$, Eq. (6.13), and $t_{s p h}$, Eq. (6.12), for the case of no particles and at zero concentration and $\phi=0.9$ are same as those for a flame with inert particles of the size 120 microns, and $\phi=0.9$. When the addition of 
inert particles increases the time interval is also increasing, which is an indication for the reduction of short acceleration happening in the finger flame scenario.

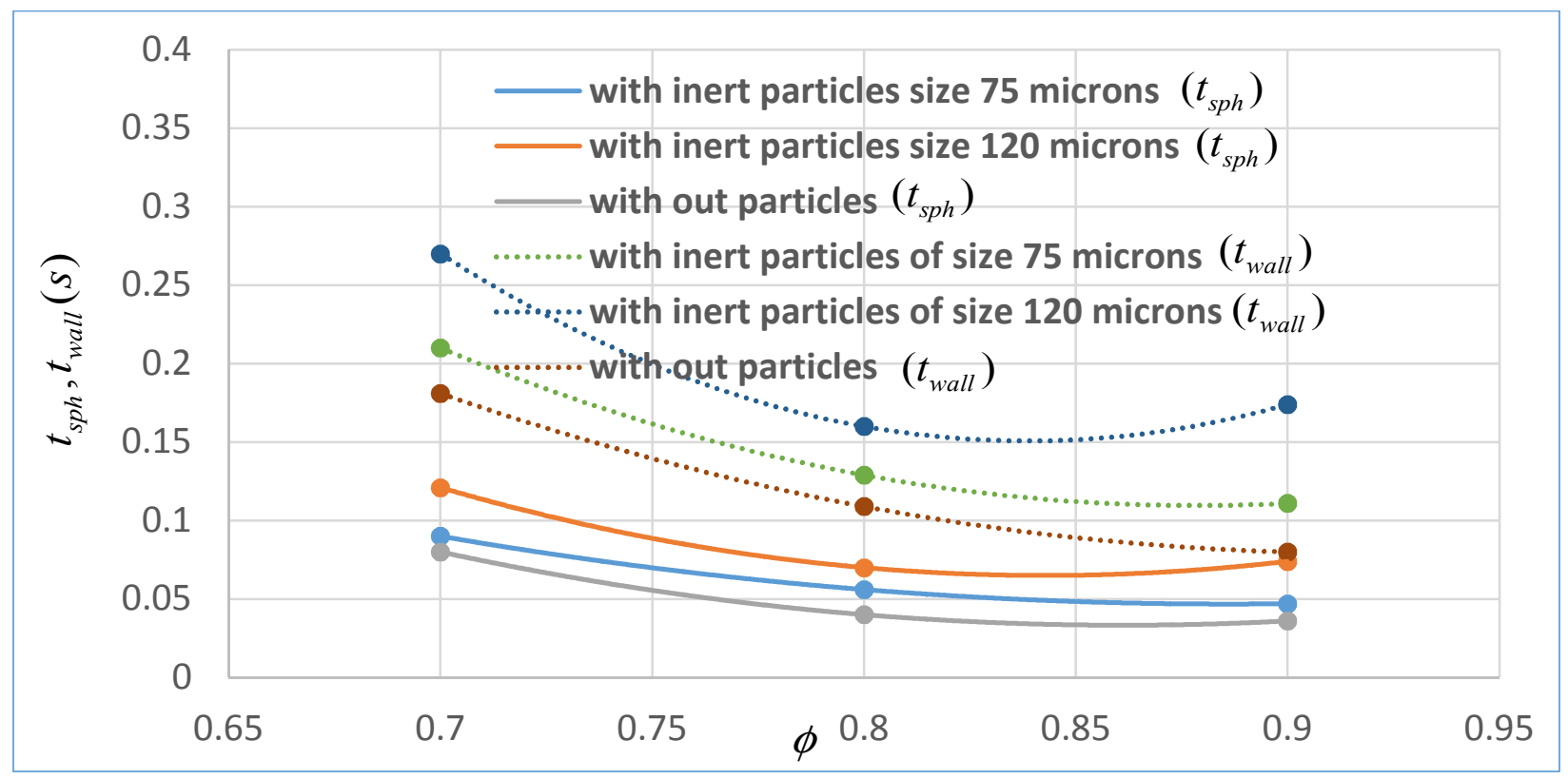

Fig 6.7 The time limitations of the finger flame acceleration, $t_{s p h}$ of Eq. (6.12), and $t_{\text {wall }}$ of Eq. (6.13), at equivalence ratio $\phi=0.7-0.9$ versus concentration of particles for methane-air flames.

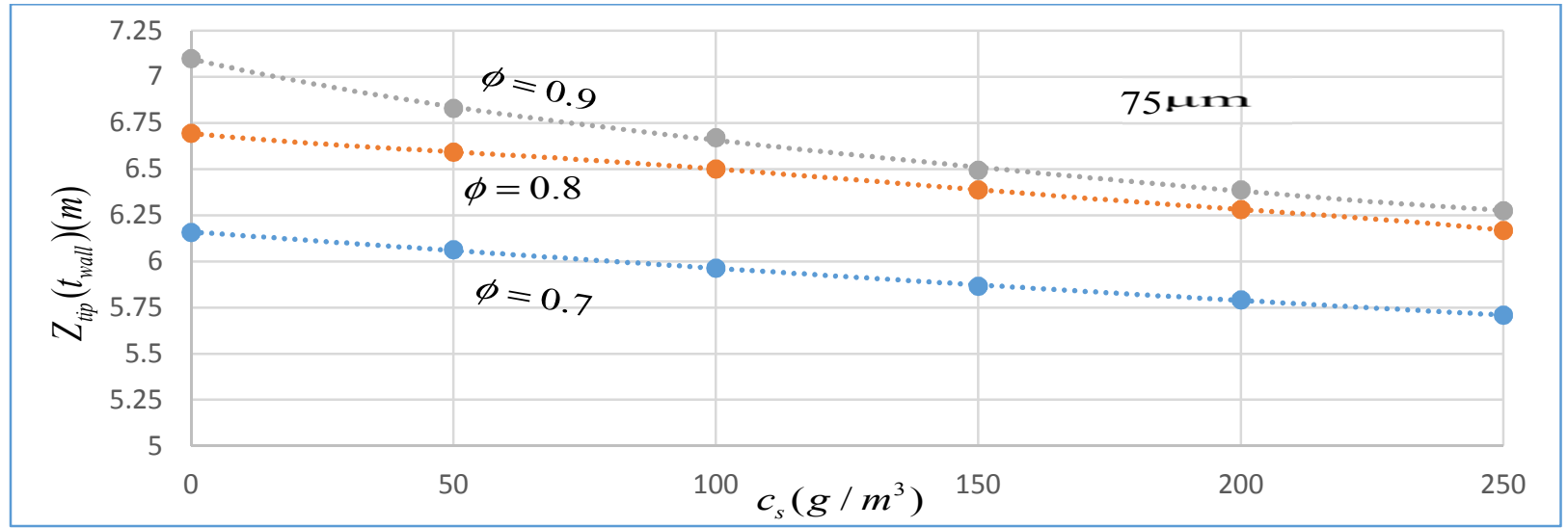

Fig 6.8: The flame tip position, Eq. (6.9), at the termination time $t_{\text {wall }}$, Eq. (6.13), versus the concentration of inert particles for fixed equivalence ratio.

The flame tip position at the instant when the flame skirt contacts the wall (which terminating the finger flame acceleration scenario) is shown in Fig. 6.4, thereby illustrating the decrease in the 
distance travelled by the flame with the increase in the concentration of particles for different equivalence ratios. This happens because the flame temperature decreases due to the heat sink effect of inert particles, which reduces the (short) period of the finger flame acceleration. Figure 6.9 shows the maximal flame tip velocity at the characteristic time, $t_{\text {wall }}$, Eq. (6.11), It is obvious that inert particles moderate the flame tip velocity with increasing dust concentration.

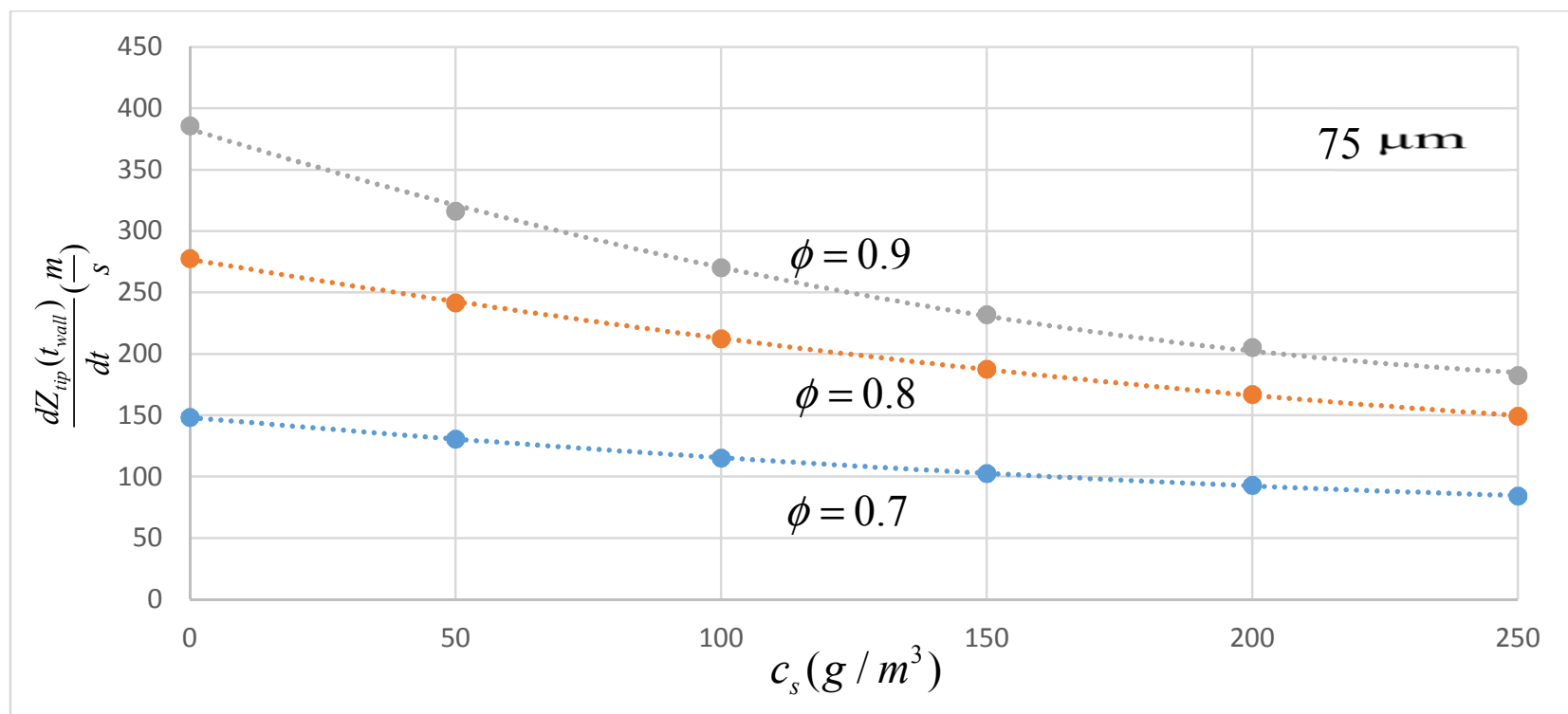

Fig. 6.9: The maximal flame tip velocity $\left(d Z_{\text {tip }} / d t\right)_{\text {wall }}$, Eq. (6.11), at the time $t_{\text {wall }}$ of Eq. (6.13) versus the concentration of inert particles for the methane-air-inert mixtures of different equivalence ratios $\phi=0.7,0.8$ and 0.9 with particles of size 75 microns.

Finally, Fig. 6.10 presents the time evolution of the flame tip velocity, Eq. (6.11), in the range $t_{s p h}<t<t_{\text {wall }}$, for a lean methane-air fuel mixture, $\phi=0.7$, with and without inert particles. It is seen, again, that the addition of inert particles moderates the flame speed in the interval $t_{s p h}<t<t_{\text {wall }}$. The plots show the same trends, namely, the flame tip velocity increases in time for methane-air flames without and with dust particles, respectively. 


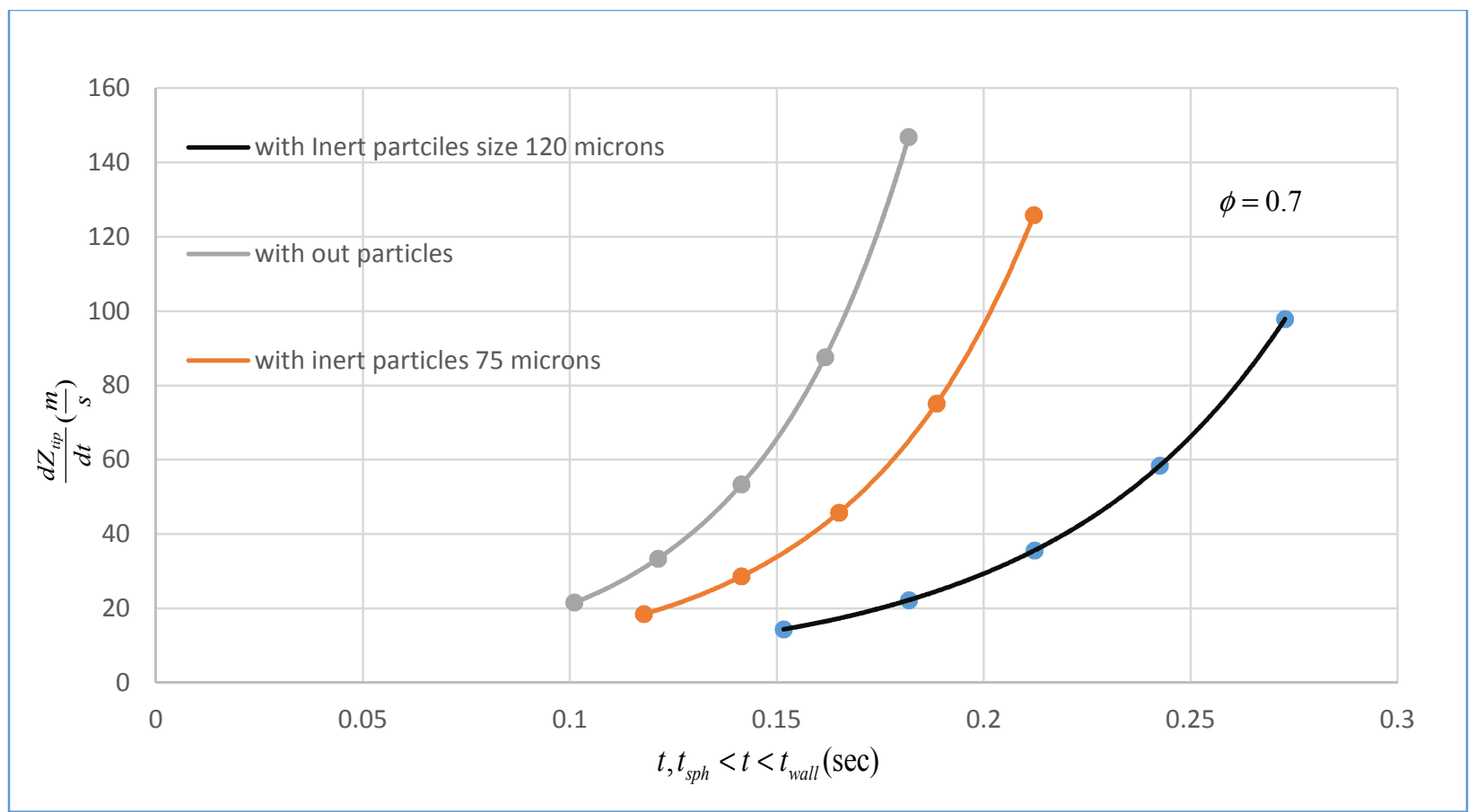

Fig. 6.10: Evolution of flame tip velocity $d Z_{t i p} / d t$, Eq. (6.10), vs the time interval between $t_{s p h}$, Eq. (6.12), and $t_{\text {wall }}$, Eq. (6.13), $t_{\text {sph }}<t<t_{\text {wall }}$. The methane-air mixture with and without inert particles for the lean fuel of equivalence ratio $\phi=0.7$.

In the above results, the effect of inert particles is illustrated with inclusion of different size of particles being 75 and 120 microns, respectively. On the other hand, in Chapter 5, the effect of combustible particles is illustrated with particle size being 25 and 75 microns. In both of these cases, the laminar flame speed increases with the presence of combustible particles, but it decreases with the presence of inert particles. Eventually, both the effects are compared in Figs. 6.11 and 6.12 for the limitations of finger flame and maximal flame tip velocities, respectively. 


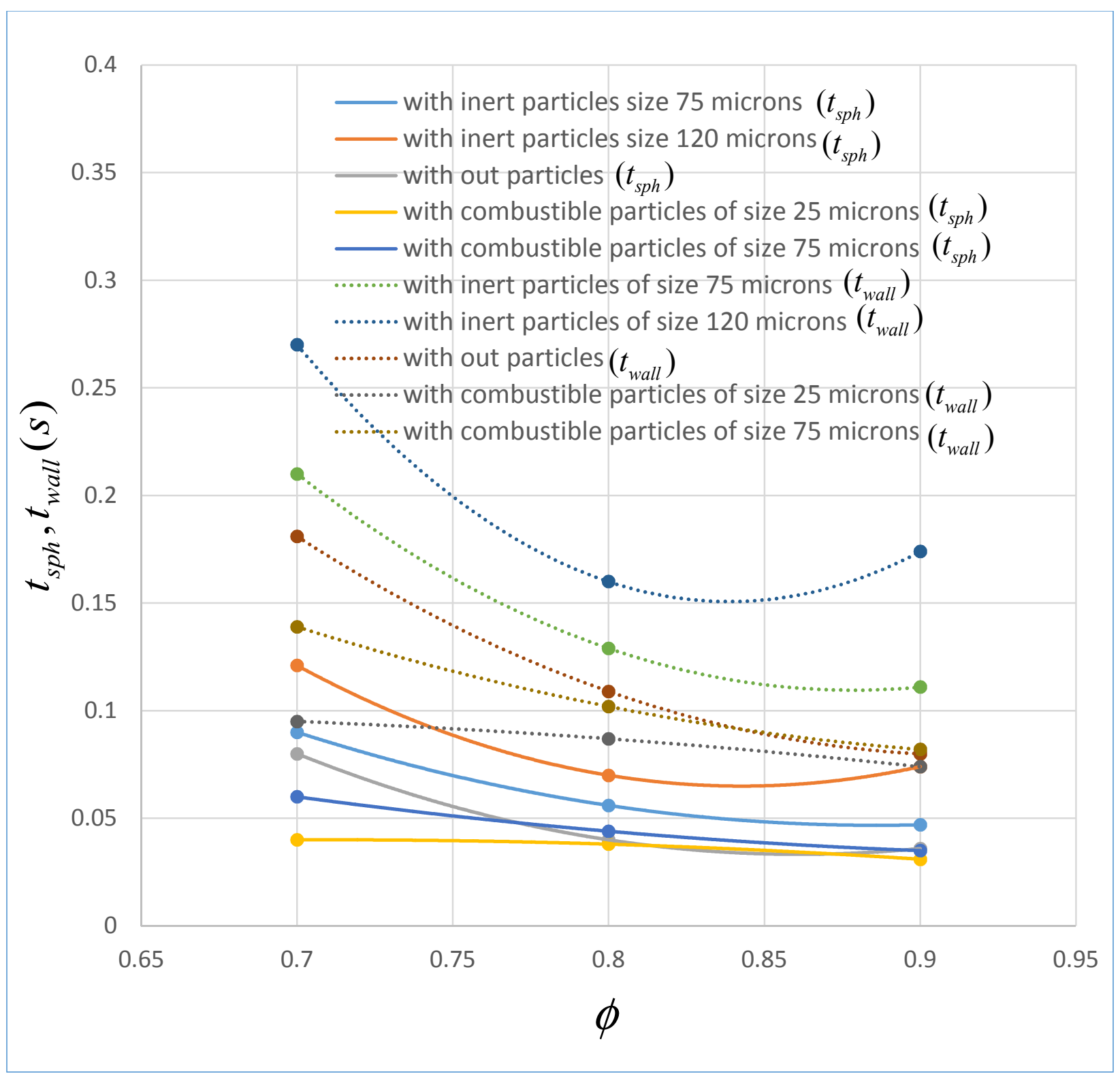

Fig 6.11 The time limitations of the finger flame acceleration, $t_{s p h}$ of Eq. (6.12) and (5.12), and $t_{\text {wall }}$ of Eq. (6.13) and (5.13), at equivalence ratio $\phi=0.7-0.9$ versus concentration of particles for methane-air flames. 


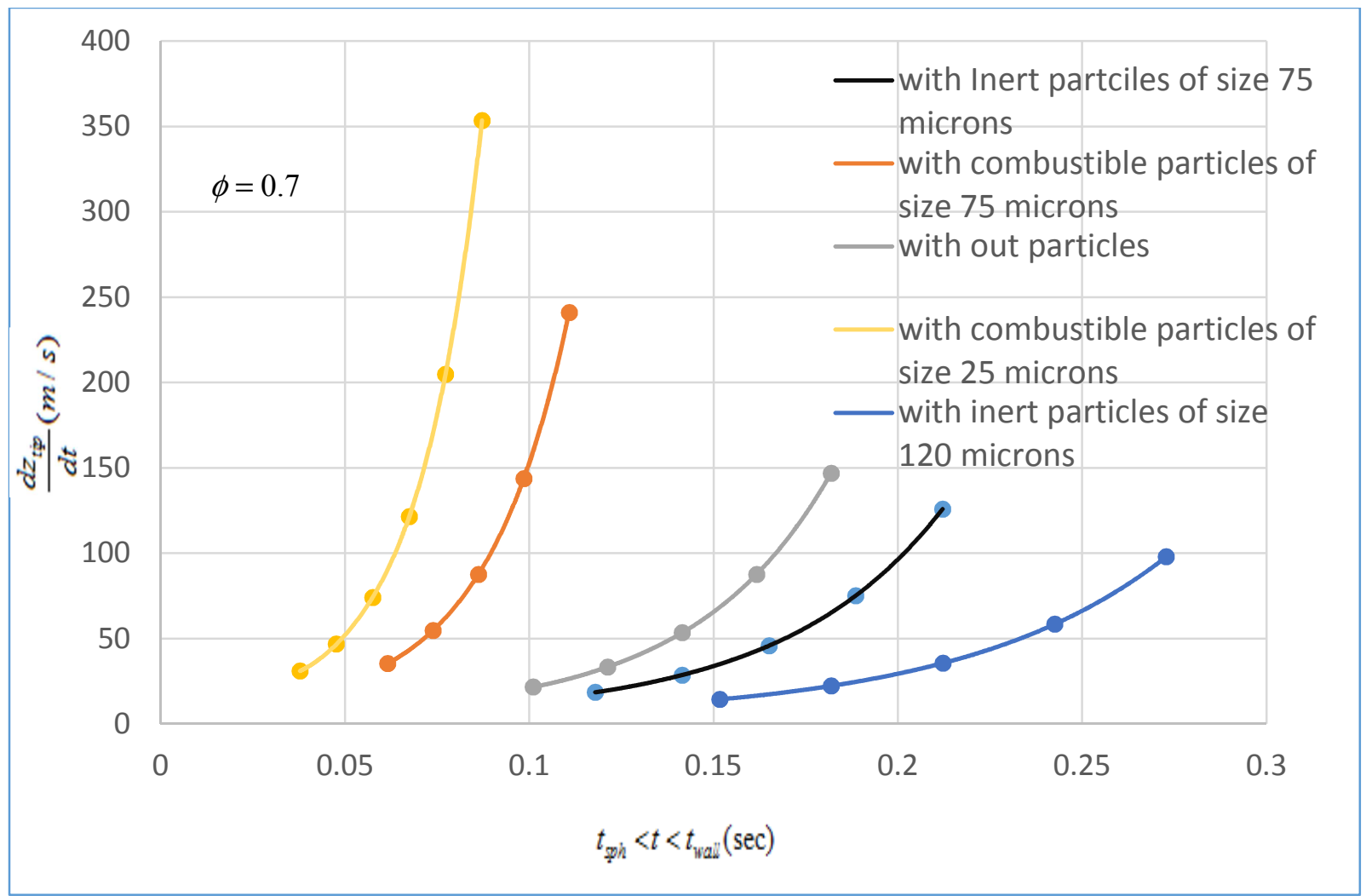

Fig. 6.12: Evolution of flame tip velocity $d Z_{t i p} / d t$, Eq. (6.10) and (5.10), vs the time interval between $t_{s p h}$, Eqs. (6.12) and (5.12), and $t_{\text {wall }}$, Eqs. (6.13) and (5.13). $t_{s p h}<t<t_{\text {wall }}$. The methane-air with inert particles, combustible and without particles mixtures are shown for the lean fuel ratio $\phi=0.7$. 


\section{Conclusion and future work}

In the present thesis a predictive quantitative scenario of an accidental fire in a gaseous or gas-dust environment of a coal mine passage is provided. Specifically, we have quantified the flame front dynamics according to the mechanisms suggested in Refs. [9-11]. When an accidental ignition occurs in a mine, first, a flame develops from a smooth spherical kennel to globally-spherical, cellular (corrugated) structure. This occurs due the development of the Darrieus-Landau flame instability, and is accompanied by self-similar flame acceleration. Subsequently, such a cellular flame converts into a finger-like shape, exhibiting a tremendous acceleration, for a short time, until the flame skirts contacts the passage sidewall. For near-stoichiometric $(0.8<\phi<1.3)$ methaneair combustion, the overall acceleration scenario may promote the total burning rate (flame speed) by two orders of magnitude, up to supersonic values. In spite of the direct disaster of such a fast fire, this may also facilitate the deflagration-to detonation transition (DDT) event, leading thereby to additional hazards to the mining personal and equipment, associated with detonative shocks.

In the present study, the influences of combustible and inert dust particles are implemented through the mathematical model based on heat sink effect along with the effect of different particle sizes and concentrations are investigated. As a result the influence of combustible particles of size 25 micron increases the flame propagation intensity, whereas increase in the size of particles gradually decreases the flame propagation speed. . On the other hand, when inert particles are considered at 75-120 microns, the burning rate decreases irrespective of a concentration being considered, which means that a flame cannot sustain. These results have better agreement with the experimental results [39]. 
We will implement spatial and temporal variations of $S$ into the formulation of chapter 3 , which is being done by author's colleagues and thereby it can extended to turbulent environment through the development and employment of a unified turbulent flame formulation. 


\section{References}

[1] Davis, S.G., Quinard, J., Searby, G., 2002, Combustion and Flame, Vol. 130, pp. 123-136.

[2] Metghalchi, M., Keck, J.C., 1982, Combust. Flame, Vol. 48, pp. 191-210.

[3] Fairweather, M., Hargrave, G.K., Ibrahim, S.S., Walker, D.G., 1999, Combust. Flame, Vol. 116, pp. 504-518.

[4] Lee, J.H.S., 1984, Ann. Rev. Fluid Mech, Vol. 16, pp. 311-336.

[5] Mark, S., Quirk, J.J., 1997, Journal of Fluid Mechanics, Vol. 339, pp. 89-119.

[6] Yang, Y., He, X.Q., 15-18 October 2003, In Proceedings of 5th International Autumn Seminar on Propellants, Explosives and Pyrotecnics, Guilin, China.

[7] Bychkov, V., Liberman, M., 2002, Phys. Fluids, Vol. 14, pp. 2024-2025.

[8] Clanet, C., Searby, G., 1996, Combust. Flame, Vol. 105, pp. 225-238.

[9] Bychkov, V., Akkerman, V., Fru, G., Petchenko, A., Eriksson, L.E., 2007, Combust. Flame, Vol. 150, pp. 263-276.

[10] Subbotin, V.A., 1998, Combust. Expl. Shock Waves, Vol. 34, pp. 77-87.

[11] Schelkin, K.I., 1940, Combust. Flame, Vol. 10, pp. 823-827.

[12] Kagan, L., Sivashinsky, G.I., 2003. Combust. Flame, Vol. 134, pp. 389-397.

[13] Ott, J.D., Oran, E.S., Anderson, J.A., 2003, AIAA Journal, Vol. 41, pp. 1391-1396.

[14] Kagan, L., Sivashinsky, G., 2010. Flow, Turb. Combust. Vol. 84(3), pp. 423-437.

[15] Zel'dovich, Y.B., Barenblatt, G.I., Librovich, V.B., Makhviladze, G.M., 1985. Mathematical Theory of Combustion and Explosion, Consultants Bureau, New York.

[16] Oran, E.S., Gamezo, V.N., 2007, Combust. Flame, Vol. 148, pp. 4-47.

[17] Smirnov, N.N., Li, P., 1995. Combust. Flame, Vol. 101, pp. 46-67.

[18] Schelkin, K.J., 1940. Exp. Theor. Phys. Vol. 10, pp. 823. 
[19] Bychkov, V., Petchenko, A., Akkerman, V., Eriksson, L.E., 2005, Phys. Rev. E, Vol. 72, 0463070.

[20] Akkerman, V., Bychkov, V., Petchenko, A., Eriksson, L.E., 2006. Combust. Flame, Vol. 145, pp. 206-219.

[21] Mardani, A., Tabejamaat, S., Mohammadi, M.B., 2011. Combust. Theory Model. Vol. 15, pp. $753-772$.

[22] Park, J.W., Oh, C.B., 2012. Int. J. Hydrogen Energy, Vol. 37, pp. 7877-7888.

[23] Essenhigh, R. H., Csaba, J., 1963. Ninth International Symposium on Combustion, The Combustion Institute, Pittsburgh, Vol. 9, pp. 111-125.

[24] Smoot, D., Horton, M., 1977, Prog. Ener. Combust. Sci. Vol. 1, pp. 235-258.

[25] Mitani, T., 1981, Combust. Flame, Vol. 43, pp. 243-253

[26] Berald, A.L., Ross, H., Facc, L., Tangirala, V., 1990. Combust Flame, Vol. 82, pp. 448-450.

[27] Berald, A.L., Tangirala, V., 1991. Prog. Astronaut. Aeronaut. Vol. 132, pp. 159-172.

[28] Seshadri, K., Berlad, A.L., Tangirala, V., 1992. Combust. Flame, Vol. 89, pp. 333-342.

[29] Law, C. K., 2006. Combustion Physics. Cambridge: Cambridge UP.

[30] Akkerman, V., Law, C.K., Bychkov, V., 2011, Phys. Rev. E Vol. 83 (2), 026305.

[31] Pelce, P., Clavin, P., 1982 .Fluid Mech. Vol. 124, pp. 219-237.

[32] Akkerman, V., Bychkov, V., 2003, Combust. Theory Modelling, Vol. 7, pp. 767 - 794.

[33] Bychkov, V., Valiev. D., Eriksson, L.E., 2008, Phys. Rev. Lett., Vol. 101, 164501.

[34] Valiev D., Bychkov, V., Akkerman, V., Law, C.K., Eriksson, L.E., 2010. Combust. Flame, Vol. 157, pp. 12-21.

[35] Suard, S., Haldenwang, P., Nicoli, C., 2004. Comptes Rendus Mecanique, Vol. 332, pp. 387396. 
[36] Solomon, P.R., Colket, M.B., 1979. Symposium (International) on Combustion, Vol. 17, pp. $131-143$.

[37] Smoot, L.D., Smith, P.J., 1985. Coal Combustion and Gasification .

[38] Morley C., Gaseq (Ver 0.79), < http://www.c.morley.dsl.pipex.com/> (2005).

[39] Rockwell, S.R., Rangwala, A.S., 2013, Fire Safety Journal, Vol. 59, pp. $22-29$. 